\title{
A COMPOSITE PLASTICITY MODEL FOR CONCRETE
}

\author{
PETER H. FEENSTRA $†$ and RENÉ DE BORST $\ddagger$ \\ Delft University of Technology, Faculty of Civil Engineering, P.O. Box 5048, 2600 GA Delft, \\ The Netherlands
}

(Received 25 March 1994; in revised form 6 March 1995)

\begin{abstract}
A composite yield function is used to describe the behavior of plain and reinforced concrete in biaxial stress under monotonic loading conditions. A Rankine yield criterion is used to monitor the in-plane tensile stresses and a Drucker Prager yield function controls the compressive stresses. A good agreement with experimental data for biaxial stress conditions in concrete can thus be obtained. The approach is particularly powerful for the numerical analysis of concrete structures, either plain or reinforced, which are predominantly in tension-compression biaxial stress states. Initiation of cracking in such areas frequently leads to brittle, uncontrollable failure (splitting cracks), which can often not be handled by existing approaches. The proposed Euler backward algorithm based on the composite yield function and enhanced by a consistent linearization of the integrated stress-strain relation for use within a Newton-Raphson method at the structural level, is extremely robust for this particular class of problems.
\end{abstract}

\section{INTRODUCTION}

The proper modeling of tension-compression biaxial stress states in plain and reinforced concrete is an outstanding issue in finite element analysis of concrete structures that is of utmost practical importance and very challenging at the same time. It is important for engineering practice, since such stress states frequently occur in critical regions and crack initiation under such stress conditions often acts as a precursor to progressive and brittle collapse of concrete structures. Examples are shear-critical beams in the case of reinforced concrete and splitting tests in the case of plain concrete structures. The proper modeling of this phenomenon is challenging because different inelastic mechanisms play a role under such stress conditions. For instance, if one adopts a crack model to bound the tensile stresses and a plasticity model to monitor the evolution of the compressive stresses, simultaneous satisfaction of a fracture criterion and a yield function must be achieved.

In the first applications of finite element analysis to the mechanical behavior of concrete a sudden drop to a zero stress level was adopted upon violation of the tensile strength (Rashid, 1968). Similarly, it was assumed that concrete lost its complete strength and stiffness when the compressive strength was exceeded. Soon, careful experiments using servo-controlled equipment revealed that this crude assumption did not resemble the real behavior of concrete, since a descending branch was observed in tension (Rüsch and Hilsdorf, 1963; Hordijk, 1991) as well as in compression (van Mier, 1984; Willam et al., 1986). For this reason "softening" stress-strain relations were adopted, in which the stress was made a descending function of the strain and the element size such that the total energy dissipation upon complete failure became independent of the finite element mesh. (Pietruszczak and Mróz, 1981; Bažant and Oh, 1983; Willam, 1984). It is recognized that this "energy" approach gives in an ill-posed boundary value problem, which tends to result in crack propagation that is biased by the grid lines of the discretization (Sluys, 1992; de Borst et al., 1993). Nevertheless, the method is used here because it is simpler than proper regularization procedures like non-local or gradient approaches [for a review of these procedures, see de Borst et al. (1993)], so it can readily be used for obtaining practical, engineering solutions. Its simplicity also bears the advantage that attention can be focused

$\uparrow$ Currently at TNO Building and Construction Research, Rijswijk, The Netherlands.

$¥$ Also at Eindhoven University of Technology, Faculty of Mechanical Engineering. 
on the difficulty that during progressive softening a fracture criterion for the tensile behavior and a yield function for the compressive softening behavior have to be met identically.

In the approach by de Borst and Nauta (1985), this issue was solved using a local procedure at integration point level in which cracking and plasticity were treated in an iterative fashion, such that during the computation of the plastic flow the cracking strain increment was treated as an initial strain increment, and subsequently the fracture strain increment was updated while freezing the computed plastic flow increment, etc. Although this procedure has been applied successfully in a number of calculations, numerical difficulties with state changes have also been reported (Crisfield and Wills, 1989), especially in plane-stress situations.

In this contribution we shall discuss a composite yield function, in which the behavior in compression as well as that in tension is modeled using a plasticity approach. A Rankine (principal stress) yield criterion is used to limit the tensile stresses and a Drucker-Prager yield contour is employed to model the compression-compression regime in biaxial stress. It turns out that this composite yield contour closely matches the classical Kupfer and Gerstle data (1973). The fact that both tensile and compressive failures are now modeled using a plasticity-based concept has the advantage that the "corner" regime, that is where both yield contours intersect, can be handled using established concepts from computational plasticity (de Borst, 1986; Simo et al., 1988). Using the Koiter (1953) generalization for plastic flow at a corner in the yield contour, a robust Euler backward integration algorithm can be devised, including the associated consistently linearized tangent stiffness matrix for use within Newton's method.

A drawback of the plasticity-based approach is that the stiffness degradation due to progressive damage is not modeled. However, experimental evidence shows that the stiffness degradation due to tensile cracking is substantial only when the tensile cracking has developed fully (Willam et al., 1986; Hordijk, 1991). The stiffness degradation due to compressive loading is even less pronounced than the stiffness degradation due to tensile loading. Since full cyclic loading is not considered in this study, only monotonic loading where only local unloading occurs, neglecting the degradation of the elastic stiffness does not seem to entail major errors.

We shall start this paper by formulating the composite yield surface. Then, we will discuss the behavior of plain concrete, thereby focusing attention on the softening behavior in tension and in compression. The equivalent uniaxial stress-strain relations for tension and compression that have been adopted are discussed. In both cases a fracture energy has been defined in order to achieve a reasonable degree of discretization insensitivity in numerical calculations. Next, the algorithmic aspects of the model are discussed and the derivation of the linearization moduli will be given. Some illustrative examples of a splitting test and reinforced shear wall panel conclude the discussion.

It is recognized that plasticity-based crack models have been advocated before [e.g. Willam and Warnke (1975); Chen and Chen (1975); Buyukozturk (1977); Murray et al. (1979)], but the present enhancements with a second fracture energy parameter that controls the (gradual) compressive softening and the robustness of the algorithm presented here are considered to be major steps forward.

\section{FORMULATION OF A COMPOSITE YIELD SURFACE}

In this study, we shall utilize a composite yield contour which bounds the admissible stress states such that a Rankine yield function, denoted as $f_{1}$, is used to model the tensiontension region and a Drucker-Prager yield function, say $f_{2}$, is employed to bound the compressive stresses. The model will be formulated in a plane-strain configuration and the plane-stress condition, $\sigma_{z z}=0$, will be enforced by applying a compression/expansion algorithm (de Borst, 1991). In this algorithm, the strain vector is expanded by including the normal strain in the constrained direction in the beginning of an iteration. Then, the constitutive model is evaluated in the expanded, or plane-strain stress space. Finally, the stress vector is compressed such that the condition $\sigma_{z z}=0$ is enforced rigorously. 
The composite yield contour is thus expressed in a plane-strain stress space with $\boldsymbol{\sigma}^{\mathrm{T}}=\left\{\sigma_{x x}, \sigma_{y y}, \sigma_{z z}, \sigma_{x y}\right\}$, by the following yield functions:

$$
\left\{\begin{array}{l}
f_{1}=\left(1 / 2 \boldsymbol{\sigma}^{\mathrm{T}} \mathbf{P}_{1} \boldsymbol{\sigma}\right)^{1 / 2}+1 / 2 \boldsymbol{\pi}^{\mathrm{T}} \boldsymbol{\sigma}-\bar{\sigma}_{1}\left(\kappa_{1}\right) \\
f_{2}=\left(1 / 2 \boldsymbol{\sigma}^{\mathrm{T}} \mathbf{P}_{2} \boldsymbol{\sigma}\right)^{1 / 2}+\alpha_{f} \boldsymbol{\pi}_{2}^{\mathrm{T}} \boldsymbol{\sigma}-\beta \bar{\sigma}_{2}\left(\kappa_{2}\right)
\end{array}\right.
$$

with the projection matrices $\mathbf{P}_{1}$ and $\mathbf{P}_{2}$ given by

$$
\mathbf{P}_{1}=\left[\begin{array}{rrrr}
1 / 2 & -1 / 2 & 0 & 0 \\
-1 / 2 & 1 / 2 & 0 & 0 \\
0 & 0 & 0 & 0 \\
0 & 0 & 0 & 2
\end{array}\right]
$$

and

$$
\mathbf{P}_{2}=\left[\begin{array}{rrrr}
2 & -1 & -1 & 0 \\
-1 & 2 & -1 & 0 \\
-1 & -1 & 2 & 0 \\
0 & 0 & 0 & 6
\end{array}\right]
$$

respectively. The projection vectors are given by

$$
\begin{aligned}
& \boldsymbol{\pi}_{1}^{\mathrm{T}}=\{1,1,0,0\} \\
& \boldsymbol{\pi}_{2}^{\mathrm{T}}=\{1,1,1,0\},
\end{aligned}
$$

respectively. The equivalent stress $\sigma_{1}$ is the uniaxial tensile strength as a function of some internal parameter, say $\kappa_{1}$. The equivalent stress $\bar{\sigma}_{2}$ is the uniaxial compressive strength, which is also expressed as a function of an internal parameter, say $\kappa_{2}$. The factor $\alpha_{f}$ can be related to the ratio between the biaxial compressive strength and the uniaxial compressive strength, $\beta_{\mathrm{c}}$,

$$
\alpha_{f}=\frac{1-\beta_{\mathrm{c}}}{1-2 \beta_{\mathrm{c}}}
$$

The factor $\beta$ is then given by

$$
\beta=\frac{\beta_{\mathrm{c}}}{2 \beta_{\mathrm{c}}-1}
$$

A comparison with the experimental data of Kupfer and Gerstle (1973) shows that the assumed yield contour with $\beta_{\mathrm{c}}=1.2$ closely matches experimental data, Fig. 1 .

Assuming small strains, we adopt the additive decomposition of the strain rate vector $\dot{\varepsilon}$ into an elastic, reversible part $\dot{\varepsilon}_{\mathrm{e}}$ and an inelastic part $\dot{\boldsymbol{\varepsilon}}_{\mathrm{c}}$,

$$
\dot{\varepsilon}=\dot{\boldsymbol{\varepsilon}}_{\mathrm{e}}+\dot{\varepsilon}_{\mathrm{c}}
$$

where the inelastic part $\dot{\boldsymbol{\varepsilon}}_{\mathrm{c}}$ can be considered as irreversible in the case that elastic stiffness degradation is neglected. The elastic strain rate determines the stress rate through the elastic stiffness matrix $\mathbf{D}_{\text {. }}$ 


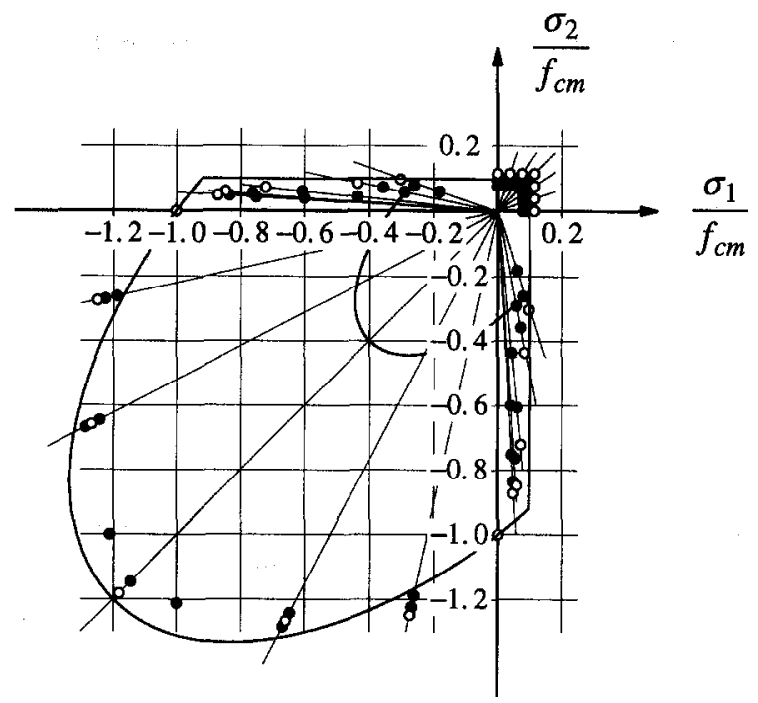

Fig. 1. Comparison of a Rankine/Drucker-Prager yield surface with experimental data of Kupfer and Gerstlc (1973).

$$
\dot{\sigma}=\mathbf{D}_{\mathrm{e}} \dot{\varepsilon}_{\mathrm{e}}
$$

The evolution of the inelastic strain rate is determined by Koiter's rule (Koiter, 1953), which allows for a summation of the inelastic strain of each yield function according to

$$
\dot{\boldsymbol{\varepsilon}}_{\mathrm{c}}=\sum_{j=1}^{2} \dot{\lambda}_{j} \partial_{\sigma} g_{j}
$$

in which the plastic potential functions $g_{j}$ are introduced. The notation $\partial_{\sigma} g_{j}$ is used to denote the derivative of the function $g_{j}$ with respect to the stress vector $\sigma$. The rate of the inelastic multipliers $\dot{\lambda}_{j}$ has to comply with the Kuhn-Tucker conditions

$$
\dot{\lambda}_{j} \geqslant 0 \quad f_{j} \leqslant 0 \quad \dot{\lambda}_{j} f_{j}=0 .
$$

In this study, the plastic potential functions are assumed to be given by

$$
\left\{\begin{array}{c}
g_{1}=f_{1} \\
g_{2}=\left(1 / 2 \boldsymbol{\sigma}^{\mathrm{T}} \mathbf{P}_{2} \boldsymbol{\sigma}\right)^{1 / 2}+\alpha_{g} \pi_{2}^{\mathrm{T}} \boldsymbol{\sigma}-\beta \bar{\sigma}_{2}\left(\kappa_{2}\right)
\end{array}\right.
$$

such that we have associated plasticity for the tensile regime and non-associated plasticity for the compressive stress regime. The factor $\alpha_{g}$ can be expressed as a function of the dilatancy angle $\psi$, according to

$$
\alpha_{g}=\frac{2 \sin \psi}{3-\sin \psi} .
$$

\section{BEHAVIOR OF PLAIN CONCRETE}

The behavior of concrete is highly non-linear due to irreversible processes in the material caused by debonding and internal cracking. This process of progressive fracturing results in a descending load-deformation relation at structural level after a limit load has been reached. To obtain an approach that is feasible for analyzing large concrete structures, a constitutive model should be formulated in a "smeared" format in which the damaged material is considered to be a continuum in which the notions of stress and strain apply. Consequently, the damage in the material should also be considered as distributed. In here, 
it is assumed that the damage can be represented by the internal parameters: $\kappa_{1}$ in tension and $\kappa_{2}$ in compression. These internal parameters can be considered as damage indicators; if $\kappa_{j}=0$ then there is no damage due to stresses in the regime bounded by the yield surface $f_{j}$ else there is damage which is implicitly related to the stress state through the yield surfaces. It is most appealing to assume that the evolution of the internal variables is given by a work-hardening hypothesis $\left(\dot{W}^{\mathrm{p}}=\sigma^{\mathrm{T}} \dot{\boldsymbol{\varepsilon}}^{\mathrm{p}}\right)$, which implies that the internal parameters are determined by the inelastic work rate for the respective yield functions, i.e.

$$
\dot{W}_{i}^{\mathrm{p}}=\sum_{j=1}^{2} \dot{\lambda}_{j} \zeta_{i j} \frac{\sigma_{i j}}{\sigma_{j}} \partial_{\sigma} g_{j} \equiv \sigma_{i}\left(\kappa_{i}\right) \dot{\kappa}_{i}
$$

with scalars $\zeta_{i j}$ which represent the coupling of the damage in the material at different stress situations. Obviously, $\zeta_{i i}$ is equal to one. With the application of Euler's theorem of homogeneous functions, eqns (11) and (13) result in the following expression for the evolution of the internal parameters

$$
\dot{\kappa}_{i}=\sum_{j=1}^{2} \zeta_{i j} \dot{\lambda}_{j}
$$

Setting

$$
\dot{\mathbf{q}}=\left\{\begin{array}{c}
\dot{\kappa}_{1} \\
\dot{\kappa}_{2}
\end{array}\right\} \quad \mathbf{h}_{1}=\left\{\begin{array}{c}
1 \\
\zeta_{21}
\end{array}\right\} \quad \mathbf{h}_{2}=\left\{\begin{array}{c}
\zeta_{12} \\
1
\end{array}\right\}
$$

eqn (14) can formally be expressed as

$$
\dot{\mathbf{q}}=\sum_{j=1}^{2} \lambda_{j} \mathbf{h}_{j}
$$

In this way, the evolution of the internal parameters is fully determined by the inelastic strain rate and can be considered as a measure of the progressive fracturing in the material. Because a work-hardening hypothesis is underlying eqn (16), it is possible to relate the total plastic work to the energy dissipation of the material.

For tensile cracking the concept of energy dissipation, the fracture energy $G_{\mathrm{f}}$, has been used extensively in finite element calculations and can be considered as accepted in the engineering community. With the assumption that the fracture energy is uniformly dissipated in a representative area of the structure, the equivalent length, the finite element calculations will lead to results that are insensitive with regard to the global structural response upon mesh refinement, at least below a certain level of refinement. The concept of released energy and equivalent length is also applied here to model the compressive softening behavior via the introduction of a compressive fracture energy $G_{\mathrm{c}}$ (Willam et al., 1986; Vonk, 1992).

In finite element calculations the equivalent length, $h$, corresponds to a representative dimension of the mesh size, as pointed out by many authors (Bažant and Oh, 1983; Willam, 1984 ; Rots, 1988; Oliver, 1989). The equivalent length at least depends on the chosen element type, element size, element shape and the integration scheme. In this study it is assumed that the equivalent length can be related to the area of an element $\left(A_{\mathrm{e}}\right)$

$$
h=\alpha_{h} A_{\mathrm{e}}^{1 / 2}=\alpha_{h}\left(\sum_{\xi=1}^{n_{\xi}} \sum_{\eta=1}^{n_{\eta}} \operatorname{det}(\mathbf{J}) w_{\xi} w_{\eta}\right)^{1 / 2}
$$

in which $w_{\xi}$ and $w_{\eta}$ are the weight factors of the Gaussian integration rule as it is assumed that the elements are integrated numerically. The local, isoparametric coordinates of the 


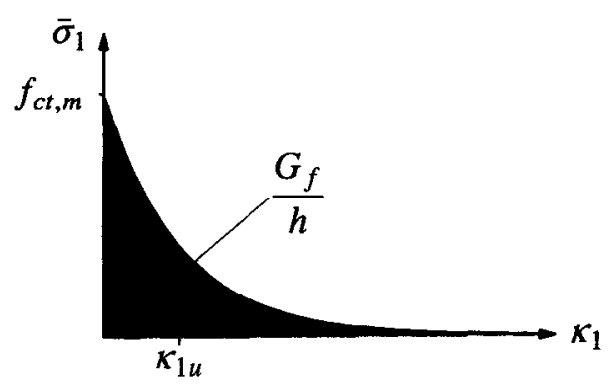

(a)

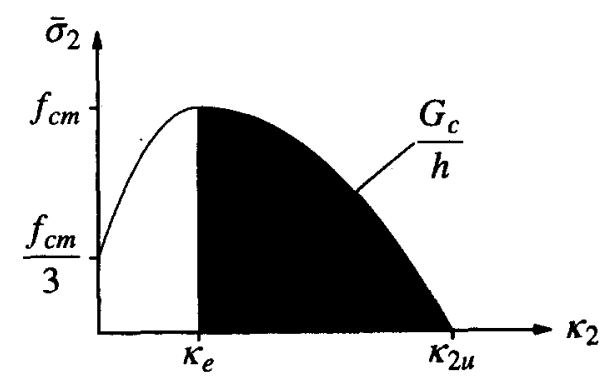

(b)

Fig. 2. Equivalent stress-equivalent strain diagrams : (a) in tension; (b) in compression.

integration points are given by $\xi$ and $\eta$, and $\operatorname{det}(\mathbf{J})$ is the Jacobian of the transformation between the local, isoparametric coordinates and the global coordinate system. The factor $\alpha_{h}$ is a modification factor which is equal to one for quadratic elements and equal to $\sqrt{ } 2$ for linear elements (Rots, 1988). For most practical applications the formulation for the equivalent length, eqn (17), gives a good approximation.

In this study a simple exponential softening diagram will be used to model the tensile stress-strain relationship, Fig. 2(a), such that the equivalent stress as a function of the internal damage parameter $\kappa_{1}$ is given by

$$
\bar{\sigma}_{1}\left(\kappa_{1}\right)=f_{\mathrm{ct}, \mathrm{m}} \exp \left(-\kappa_{1} / \kappa_{1 \mathrm{u}}\right)
$$

and the ultimate damage parameter $\kappa_{1 \mathrm{u}}$ reads

$$
\kappa_{\mathrm{lu}}=\frac{G_{\mathrm{f}}}{h f_{\mathrm{ct}, \mathrm{m}}},
$$

with $f_{\mathrm{ct}, \mathrm{m}}$ the tensile strength of concrete.

The behavior in compression will be modeled with a compression softening model as defined by the following parabolic equivalent stress-equivalent strain diagram, see Fig. 2(b),

$$
\bar{\sigma}_{2}\left(\kappa_{2}\right)=\left\{\begin{array}{c}
\frac{f_{\mathrm{cm}}}{3}\left(1+4 \frac{\kappa_{2}}{\kappa_{\mathrm{e}}}-2 \frac{\kappa_{2}^{2}}{\kappa_{\mathrm{e}}^{2}}\right) \text { if } \kappa_{2}<\kappa_{\mathrm{e}} \\
f_{\mathrm{cm}}\left(1-\frac{\left(\kappa_{2}-\kappa_{\mathrm{e}}\right)^{2}}{\left(\kappa_{2 \mathrm{u}}-\kappa_{\mathrm{e}}\right)^{2}}\right) \text { if } \quad \kappa_{\mathrm{e}} \leqslant \kappa_{2}<\kappa_{2 \mathrm{u}}
\end{array}\right.
$$

with $f_{\mathrm{cm}}$ the mean value of the compressive strength. The maximum compressive strength will be reached at an equivalent strain $\kappa_{2}=\kappa_{\mathrm{e}}$ which is independent of the element size $h$ and reads 


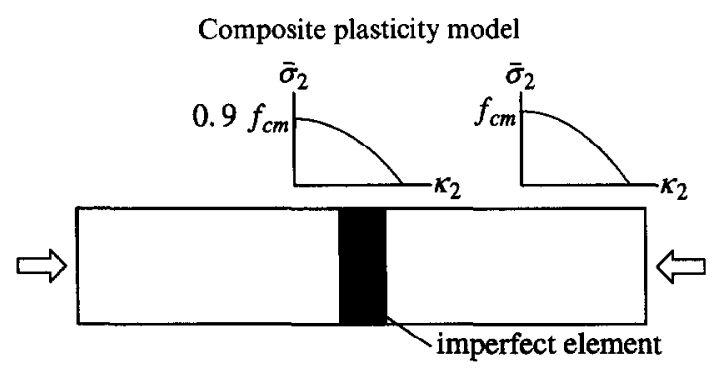

Fig. 3. Simple compression bar with one imperfect element.

$$
\kappa_{\mathrm{e}}=\frac{4 f_{\mathrm{cm}}}{3 E_{\mathrm{c}}}
$$

where $E_{\mathrm{c}}$ is the Young's modulus of the concrete. The maximum equivalent strain $\kappa_{2 \mathrm{u}}$ is related to the compressive fracture energy $G_{\mathrm{c}}$ and the element size $h$ and reads

$$
\kappa_{2 \mathrm{u}}=\kappa_{\mathrm{e}}+1.5 \frac{G_{\mathrm{c}}}{h f_{\mathrm{cm}}}
$$

The compressive fracture energy $G_{\mathrm{c}}$ is assumed to be a material parameter for which experimental evidence has been provided by Vonk (1992).

To demonstrate the mesh insensitivity of the structural response when a compressive fracture energy is supplied to the model, a simple bar loaded in compression is presented. The case is similar to the problem of a simple bar loaded in tension (Crisfield, 1982). Consider the bar of Fig. 3 which is divided into $n$ elements with $n=10,15,20$ and 25. The following material properties have been assigned: Young's modulus $E_{\mathrm{c}}=30,000 \mathrm{MPa}$, Poisson's ratio $v=0.2, \bar{\sigma}_{2}=3.0 \mathrm{MPa}$ and a compressive fracture energy $G_{\mathrm{c}}=1 \mathrm{~N} \mathrm{~mm}^{-1}$. One element in the center of the bar has a slight imperfection to trigger the localization: $\bar{\sigma}_{2}=2.7 \mathrm{MPa}$ and a compressive fracture energy $G_{\mathrm{c}}=0.9 \mathrm{~N} \mathrm{~mm}^{-1}$. The other material parameters remain the same. The load-displacement response of the bar is depicted in Fig. 4(a) for the energy-based regularization method. It is observed from Fig. 4 that the response is almost independent of the number of elements. The response of the structure with a constitutive model in which the compressive fracture energy $G_{\mathrm{c}}$ does not enter as a separate material parameter, Fig. 4(b), shows a severe mesh-dependent behavior in the post-peak regime: the response becomes more brittle with an increasing number of elements.

We conclude this section with a discussion on the coupling between damage due to compressive loading and damage due to tensile loading. Considering the Kupfer and Gerstle data, Fig. 1, we observe a reduction of the compressive strength under increasing lateral compression. A large number of the plasticity models which have been proposed previously set out to obtain an exact fit of these biaxial data. However, compressive loading results in fracturing of the material [e.g. van Mier (1984); Vonk (1992)], which obviously reduces the tensile strength in the lateral direction. This can be observed in Fig. 1 where the initial yield surface of Drucker-Prager at an equivalent stress equal to one-third of the maximum compressive stress is also plotted. If the material is loaded beyond this initial yield surface and if a coupling as in eqn (16) is present, then the equivalent stress for the Rankine condition will be reduced even if the tensile stress does not violate the Rankine yield condition. In fact, not only the maximum tensile strength will be reduced but the model also provides for a reduction of the tensile fracture energy due to lateral compressive loading. Because experimental evidence is scarce, the coupling will be neglected in this study by assuming that $\zeta_{12}=\zeta_{21}=0$.

\section{ALGORITHMIC ASPECTS OF THE CONSTITUTIVE MODEL}

The evolution equations in the flow theory of plasticity can be regarded as strain driven in the sense that the total strain vector, the inelastic strain vector and the internal variables 


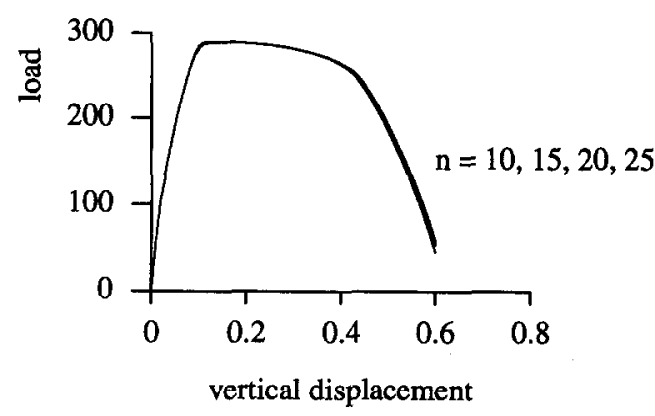

(a)

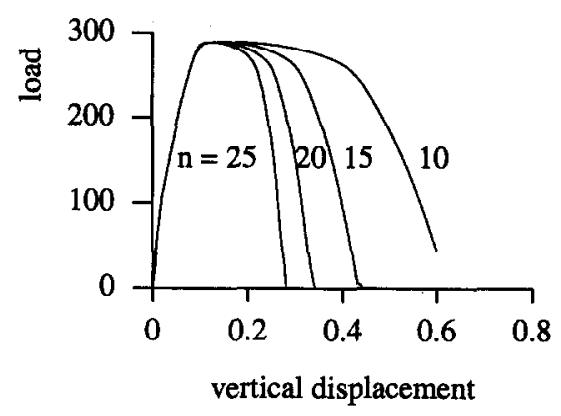

(b)

Fig. 4. Load-displacement diagrams for a simple compression bar: (a) with energy approach ; (b) without energy approach

are known at time $t$, and that the incremental strain vector $\Delta \varepsilon^{(i+1)}$ in the current iteration $(i+1)$ follows from the loading regime. The basic problem in computational elasto-plasticity is that the elasto-plastic constitutive equations have to be updated in a consistent manner

$$
\left({ }^{t} \boldsymbol{\sigma},{ }^{\prime} \boldsymbol{\varepsilon},{ }^{t} \mathbf{q} ; \Delta \varepsilon^{(i+1)}\right) \rightarrow\left(\sigma^{(i+1)}, \boldsymbol{\varepsilon}^{(i+1)}, \mathbf{q}^{(i+1)}\right) .
$$

By applying the fully implicit Euler backward algorithm this problem is transformed into a constrained optimization problem governed by discrete Kuhn-Tucker conditions (Simo et al., 1988). Even when the yield surface is highly distorted the Euler backward algorithm is unconditionally stable and accurate (Ortiz and Popov, 1985; Schellekens and de Borst, 1991) and is therefore well suited for the constitutive model in this study. Application of the Euler backward algorithm results in a discrete set of equations

$$
\begin{aligned}
& \boldsymbol{\varepsilon}^{(i+1)}={ }^{t} \boldsymbol{\varepsilon}+\Delta \varepsilon^{(i+1)} \\
& \boldsymbol{\sigma}^{(i+1)}=\mathbf{D}_{\mathrm{e}}\left(\varepsilon^{(i+1)}-\varepsilon_{\mathrm{c}}^{(i+1)}\right) \\
& \boldsymbol{\varepsilon}_{\mathrm{c}}^{(i+1)}={ }^{t} \boldsymbol{\varepsilon}_{\mathrm{c}}+\sum_{j=1}^{2} \Delta \lambda_{j}^{(i+1)} \partial_{\sigma} g_{j}^{(i+1)} \\
& \mathbf{q}^{(i+1)}={ }^{t} \mathbf{q}+\sum_{j=1}^{2} \Delta \lambda_{j}^{(i+1)} \mathbf{h}_{j}
\end{aligned}
$$

subject to the discrete Kuhn-Tucker conditions

$$
\Delta \lambda_{j}^{(i+1)} \geqslant 0 \quad f_{j}^{(i+1)} \leqslant 0 \quad \Delta \lambda_{j}^{(i+1)} f_{j}^{(i+1)}=0 .
$$

Because the algorithm is considered within an elastic predictor-plastic corrector algorithm an elastic trial state is introduced as 


$$
\begin{aligned}
& \boldsymbol{\varepsilon}^{\mathrm{E}}={ }^{t} \boldsymbol{\varepsilon}_{\mathrm{e}}+\Delta \boldsymbol{\varepsilon}^{(i+1)} \\
& \boldsymbol{\sigma}^{\mathrm{E}}=\mathbf{D}_{\mathrm{e}} \boldsymbol{\varepsilon}^{\mathrm{E}} \\
& \mathbf{q}^{\mathrm{E}}={ }^{t} \mathbf{q}
\end{aligned}
$$

which can be obtained by freezing inelastic flow during the time step. The Euler backward procedure now evolves as

$$
\left\{\begin{array}{c}
\boldsymbol{\sigma}^{(i+1)}=\boldsymbol{\sigma}^{\mathrm{E}}-\sum_{j=1}^{2} \Delta \lambda_{j}^{(i+1)} \mathbf{D}_{\mathrm{e}} \partial_{\sigma} g_{j}^{(i+1)} \\
\mathbf{q}^{(i+1)}=\mathbf{q}^{\mathrm{E}}+\sum_{j=1}^{2} \Delta \lambda_{j}^{(i+1)} \mathbf{h}_{j}
\end{array} .\right.
$$

For the choice of $g_{j}$ as laid down in eqn (11), eqn (26) can be elaborated as

$$
\left\{\begin{aligned}
\boldsymbol{\sigma}^{(i+1)}= & \mathbf{A}^{-1}\left\{\boldsymbol{\sigma}^{\mathrm{E}}-1 / 2 \Delta \lambda_{1}^{(i+1)} \mathbf{D}_{\mathrm{e}} \boldsymbol{\pi}_{1}-\alpha_{g} \Delta \lambda_{2}^{(i+1)} \mathbf{D}_{\mathrm{e}} \pi_{2}\right\} \\
& \mathbf{q}^{(i+1)}=\mathbf{q}^{\mathrm{E}}+\Delta \lambda_{1}^{(i+1)} \mathbf{h}_{1}+\Delta \lambda_{2}^{(i+1)} \mathbf{h}_{2}
\end{aligned}\right.
$$

with the matrix $\mathbf{A}$ given by

$$
\mathbf{A}=\mathbf{I}+\frac{\Delta \lambda_{1}^{(i+1)}}{2 \Psi_{1}} \mathbf{D}_{\mathbf{c}} \mathbf{P}_{1}+\frac{\Delta \lambda_{2}^{(i+1)}}{2 \Psi_{2}} \mathbf{D}_{\mathbf{e}} \mathbf{P}_{2}
$$

The denominators $\Psi_{1}$ and $\Psi_{2}$ which occur in eqn (28) read

$$
\left\{\begin{array}{l}
\Psi_{1}=\bar{\sigma}_{1}\left(\kappa_{1}^{(i+1)}\right)-1 / 2 \pi_{1}^{\mathrm{T}} \sigma^{(i+1)} \\
\Psi_{2}=\bar{\sigma}_{2}\left(\kappa_{2}^{(i+1)}\right)-\alpha_{f} \pi_{2}^{\mathrm{T}} \sigma^{(i+1)}
\end{array}\right.
$$

These expressions are not convenient because the updated stress $\left(\sigma^{(i+1)}, \mathbf{q}^{(i+1)}\right)$ cannot be related linearly to the trial state $\left(\sigma^{\mathrm{E}}, \mathbf{q}^{\mathrm{F}}\right)$. To obtain a more suitable form we first multiply $\sigma^{(i+1)}$ with $\pi_{2}^{\mathrm{T}} \mathbf{A}$ (de Borst, 1993) so that

$$
\boldsymbol{\pi}_{2}^{\mathrm{T}} \boldsymbol{\sigma}^{(i+1)}=\boldsymbol{\pi}_{2}^{\mathrm{T}} \boldsymbol{\sigma}^{\mathrm{E}}-1 / 2 \Delta \lambda_{1}^{(i+1)} \boldsymbol{\pi}_{2}^{\mathrm{T}} \mathbf{D}_{\mathrm{e}} \boldsymbol{\pi}_{1}-\alpha_{g} \Delta \lambda_{2}^{(i+1)} \boldsymbol{\pi}_{2}^{\mathrm{T}} \mathbf{D}_{\mathrm{e}} \boldsymbol{\pi}_{2},
$$

where the identities $\pi_{2}^{\mathrm{T}} \mathbf{D}_{\mathrm{e}} \mathbf{P}_{1}=\mathbf{0}^{\mathrm{T}}$ and $\boldsymbol{\pi}_{2}^{\mathrm{T}} \mathbf{D}_{\mathrm{e}} \mathbf{P}_{2}=\mathbf{0}^{\mathrm{T}}$ have been used, which follow from the tacit assumption of isotropic elasticity. By the same argument $\pi_{2}^{\mathrm{T}} \mathbf{D}_{\mathrm{e}} \pi_{1}=E /(1-2 v)$ and $\pi_{2}^{\mathrm{T}} \mathrm{D}_{\mathrm{e}} \pi_{2}=3 E /(1-2 v)$, so that the denominator $\Psi_{2}$ can be expressed solely in terms of the trial state variables and the inelastic multipliers

$$
\Psi_{2}=\bar{\sigma}_{2}\left(\kappa_{2}^{(i+1)}\right)-\alpha_{f} \pi_{2}^{\mathrm{T}} \sigma^{\mathrm{E}}+\frac{\alpha_{f} E}{(1-2 v)}\left(\Delta \lambda_{1}^{(i+1)}+3 \alpha_{g} \Delta \lambda_{2}^{(i+1)}\right)
$$

Next, $\Psi_{1}$ is determined by premultiplication of $\sigma^{(i+1)}$ with $\pi_{1}^{\mathrm{T}} A$, so that

$$
\boldsymbol{\pi}_{1}^{\mathrm{T}} \boldsymbol{\sigma}^{(i+1)}=\boldsymbol{\pi}_{1}^{\mathrm{T}} \boldsymbol{\sigma}^{\mathrm{E}}-1 / 2 \Delta \lambda_{1}^{(i+1)} \boldsymbol{\pi}_{1}^{\mathrm{T}} \mathbf{D}_{\mathrm{e}} \pi_{1}-\alpha_{g} \Delta \lambda_{2}^{(i+1)} \boldsymbol{\pi}_{1}^{\mathrm{T}} \mathbf{D}_{\mathrm{e}} \pi_{2}-\frac{\Delta \lambda_{2}^{(i+1)}}{2 \Psi_{2}} \pi_{1}^{\mathrm{T}} \mathbf{D}_{\mathrm{e}} \mathbf{P}_{2} \boldsymbol{\sigma}^{(i+1)}
$$

since $\boldsymbol{\pi}_{1}^{\mathrm{T}} \mathbf{D}_{\mathrm{e}} \mathbf{P}_{1}=\mathbf{0}^{\mathrm{T}}$. However, the factor $\boldsymbol{\pi}_{1}^{\mathrm{T}} \boldsymbol{\sigma}$ is still a function of the final stress because $\boldsymbol{\pi}_{1}^{\mathrm{T}} \mathbf{D}_{\mathrm{e}} \mathbf{P}_{2}=2 G\{1,1,-2,0\}$ with the shear modulus $G=E / 2(1+\nu)$. Substitution of this relation into eqn (32) results in 


$$
\begin{aligned}
\boldsymbol{\pi}_{1}^{\mathrm{T}} \boldsymbol{\sigma}^{(i+1)}=\boldsymbol{\pi}_{1}^{\mathrm{T}} \boldsymbol{\sigma}^{\mathrm{E}}-1 / 2 \Delta \lambda_{1}^{(i+1)} & \boldsymbol{\pi}_{1}^{\mathrm{T}} \mathbf{D}_{\mathrm{e}} \boldsymbol{\pi}_{1} \\
& \quad-\alpha_{g} \Delta \lambda_{2}^{(i+1)} \boldsymbol{\pi}_{1}^{\mathrm{T}} \mathbf{D}_{\mathrm{e}} \pi_{2}-\frac{\Delta \lambda_{2}^{(i+1)} G}{\Psi_{2}} \pi_{1}^{\mathrm{T}} \boldsymbol{\sigma}^{(i+1)}+\frac{2 \Delta \lambda_{2}^{(i+1)} G}{\Psi_{2}} \sigma_{z z}^{(i+1)} .
\end{aligned}
$$

The return-mapping of the stress in the $z$-direction is given by

$$
\sigma_{z 2}^{(i+1)}=\sigma_{z z, \mathrm{E}}-\frac{\Delta \lambda_{1}^{(i+1)} v E}{(1+v)(1-2 v)}-\frac{\alpha_{g} \Delta \lambda_{2}^{(i+1)} E}{(1-2 v)}+\frac{\Delta \lambda_{2}^{(i+1)} G}{\Psi_{2}} \pi_{1}^{\mathrm{T}} \sigma^{(i+1)}-\frac{2 \Delta \lambda_{2}^{(i+1)} G}{\Psi_{2}} \sigma_{z z}^{(i+1)}
$$

The factor $\pi_{1}^{\top} \sigma^{(i+1)}$ is finally obtained by substituting eqn (34) into eqn (33) which results in

$$
\begin{aligned}
\pi_{1}^{\mathrm{T}} \boldsymbol{\sigma}^{(i+1)}=\frac{\Psi_{2}+2 \Delta \lambda_{2}^{(i+1)} G}{\Psi_{2}+3 \Delta \lambda_{2}^{(i+1)} G}\left(\pi_{1}^{\mathrm{T}} \sigma^{\mathrm{E}}-\frac{\Delta \lambda_{1}^{(i+1)} E}{(1+v)(1-2 v)}-\frac{2 \alpha_{g} \Delta \lambda_{2}^{(i+1)} E}{(1-2 v)}\right) \\
+\frac{2 \Delta \lambda_{2}^{(i+1)} G}{\Psi_{2}+3 \Delta \lambda_{2}^{(i+1)} G}\left(\sigma_{z z, \mathrm{E}}-\frac{\Delta \lambda_{1}^{(i+1)} v E}{(1+v)(1-2 v)}-\frac{\alpha_{y} \Delta \lambda_{2}^{(i+1)} E}{(1-2 v)}\right) .
\end{aligned}
$$

With this result the denominators are expressed solely in terms of the trial state variables and the inelastic multipliers.

Upon substitution of $\boldsymbol{\sigma}^{(i+1)}$ and $\mathbf{q}^{(i+1)}$ as given in eqn (27), the yield functions are expressed solely in terms of the inelastic multipliers $\Delta \lambda_{j}$. The non-linear constraint equations, $f_{j}\left(\boldsymbol{\sigma}^{(i+1)}, \mathbf{q}^{(i+1)}\right)$, now reduce to a system of scalar equations according to

$$
\left\{\begin{array}{l}
f_{1}\left(\Delta \lambda_{1}^{(i+1)}, \Delta \lambda_{2}^{(i+1)}\right)=0 \\
f_{2}\left(\Delta \lambda_{1}^{(i+1)}, \Delta \lambda_{2}^{(i+1)}\right)=0
\end{array}\right.
$$

which have to be solved to obtain the final stress state with the additional constraints of the discrete Kuhn-Tucker conditions, eqns (24).

In softening plasticity, i.e. with negative hardening moduli, it is complicated to identify in the trial state which yield surfaces are active in the final state. The location of the intersection between the two yield surfaces is unknown in the beginning of the step and the initial configuration cannot provide a sufficient criterion for determining which surface is active at the end of the time step. Simo et al. (1988) have proposed an algorithm in which the assumption is made that the number of active yield surfaces in the final stress state is less than or equal to the number of active yield surfaces in the trial stress state. This implies that it is not possible that a yield function, in the trial state inactive, becomes active during the return-mapping. As explained by Pramono and Willam (1989), this assumption is not valid for softening plasticity. In this study, the approach of Simo et al. (1988) has been modified to account for the fact that a yield surface can become active during the returnmapping. Additional constraints $c_{j}$ are introduced, which indicate the status of the yield functions. Initially the constraints are determined by the violation of the yield criterion in the trial state, i.e.

$$
c_{j}=\left\{\begin{array}{lll}
1 & \text { if } & f_{j}\left(\boldsymbol{\sigma}^{\mathrm{E}}, \mathbf{q}^{\mathrm{E}}\right)>0 \\
0 & \text { if } & f_{j}\left(\boldsymbol{\sigma}^{\mathrm{E}}, \mathbf{q}^{\mathrm{E}}\right) \leqslant 0
\end{array}\right.
$$

During the return-mapping the active yield functions are determined with conditions 


$$
c_{j}=\left\{\begin{array}{lll}
1 & \text { if } & \Delta \lambda_{j}^{(i+1)}>0 \vee f_{j}>0 \\
0 & \text { if } & \Delta \lambda_{j}^{(i+1)}<0 \wedge f_{j}<0
\end{array} .\right.
$$

These additional constraints are merely introduced for numerical convenience because the non-linear constraint equations, eqns (36), are now expressed as

$$
\left\{\begin{array}{l}
c_{1} f_{1}\left(\Delta \lambda_{1}^{(i+1)}, \Delta \lambda_{2}^{(i+1)}\right)+\left(1-c_{1}\right) \Delta \lambda_{1}^{(i+1)}=0 \\
c_{2} f_{2}\left(\Delta \lambda_{1}^{(i+1)}, \Delta \lambda_{2}^{(i+1)}\right)+\left(1-c_{2}\right) \Delta \lambda_{2}^{(i+1)}=0
\end{array}\right.
$$

so that the first two conditions of eqn (24) are enforced simultaneously. The solution of this system of equations is obtained using a local Newton-Raphson iteration with a Broyden update of the Jacobian [e.g. Dennis and Schnabel (1983)]. The success of this approach depends on the initial Jacobian, which is determined here from the linearization of the yield functions in the trial state which reads

$$
J_{i j}^{(0)}=-\partial_{\sigma} f_{i}^{\mathrm{T}} \mathbf{D}_{\mathrm{e}} \partial_{\sigma} f_{j}+\partial_{q} f_{i}^{\mathrm{T}} \mathbf{h}_{j} .
$$

With this algorithm it is possible to return to a single active yield surface within a maximum of approximately 10 iterations in most cases, even if in the trial state more yield conditions are violated.

The singular point at the apex in both the Rankine and the Drucker-Prager yield surfaces is prone to numerical errors because it is easily verified that the denominators $\Psi_{1}$ as well as $\Psi_{2}$, see eqn (29), are equal to zero at the apex of the yield surfaces. In the remainder of this section the analytical solution of the stress update at the singularities will be given to show the correct limit behavior of the algorithm. Because of the assumption of isotropic elasticity, it appears that the elastic stiffness matrix $\mathbf{D}_{\mathbf{e}}$ and the projection matrices $\mathbf{P}_{1}$ and $\mathbf{P}_{2}$ have the same eigenvector space. This means that the spectral decomposition is given by the same transformation matrix, according to

$$
\begin{aligned}
& \mathbf{D}_{\mathrm{e}}=\mathbf{Q} \mathbf{\Lambda}_{\mathbf{D}} \mathbf{Q}^{\mathbf{T}} \\
& \mathbf{P}_{1}=\mathbf{Q} \mathbf{\Lambda}_{\mathbf{P}_{1}} \mathbf{Q}^{\mathbf{T}} \\
& \mathbf{P}_{2}=\mathbf{Q} \mathbf{\Lambda}_{\mathbf{P}_{2}} \mathbf{Q}^{\mathbf{T}}
\end{aligned}
$$

with the diagonal matrices

$$
\begin{aligned}
\Lambda_{\mathrm{D}} & =\operatorname{diag}\left[\frac{E}{(1+v)}, \frac{E}{(1+v)}, \frac{E}{(1-2 v)}, \frac{E}{2(1+v)}\right] \\
\boldsymbol{\Lambda}_{\mathbf{P}_{1}} & =\operatorname{diag}[0,1,0,2] \\
\Lambda_{\mathbf{P}_{2}} & =\operatorname{diag}[3,3,0,6]
\end{aligned}
$$

and the orthogonal matrix

$$
\mathbf{Q}=\left[\begin{array}{cccc}
\frac{1}{\sqrt{ } 6} & \frac{-1}{\sqrt{ } 2} & \frac{1}{\sqrt{ } 3} & 0 \\
\frac{1}{\sqrt{ } 6} & \frac{1}{\sqrt{ } 2} & \frac{1}{\sqrt{ } 3} & 0 \\
\frac{-2}{\sqrt{ } 6} & 0 & \frac{1}{\sqrt{ } 3} & 0 \\
0 & 0 & 0 & 1
\end{array}\right]
$$

which satisfies $\mathbf{Q}^{-1}=\mathbf{Q}^{\mathbf{T}}$. The matrix $\mathbf{A}$ now simplifies to 


$$
\mathbf{A}=\mathbf{Q}\left[\mathbf{I}+\frac{\Delta \lambda_{1}^{(i+1)}}{2 \Psi_{1}} \boldsymbol{\Lambda}_{\mathrm{D}} \boldsymbol{\Lambda}_{\mathrm{P}_{1}}+\frac{\Delta \lambda_{2}^{(i+1)}}{2 \Psi_{2}} \boldsymbol{\Lambda}_{\mathrm{D}} \boldsymbol{\Lambda}_{\mathrm{P}_{2}}\right] \mathbf{Q}^{\mathrm{T}}
$$

which is easily inverted as :

$\mathbf{A}^{-1}=\mathbf{Q}$

$$
\left[\begin{array}{cccc}
\frac{\Psi_{2}}{\Psi_{2}+3 \Delta \lambda_{2} G} & & \\
& \frac{\Psi_{1} \Psi_{2}}{\Psi_{1} \Psi_{2}+\Psi_{2} \Delta \lambda_{1} G+3 \Psi_{1} \Delta \lambda_{2} G} & \\
& 1 & \\
& & \frac{\Psi_{1} \Psi_{2}+\Psi_{2} \Delta \lambda_{1} G+3 \Psi_{1} \Delta \lambda_{2} G}{}
\end{array}\right] \mathbf{Q}^{\mathrm{T}}
$$

The first situation in which a singularity will be encounlered is when the final stress is at the apex of the Rankine yield function, i.e. $\sigma^{\mathrm{T}}=\left\{\bar{\sigma}_{1}, \bar{\sigma}_{1}, 0,0\right\}$. It is easily verified that if $\Psi_{1}$ and $\Delta \lambda_{2}$ equal zero, the limit of the mapping matrix is given by

$$
\lim _{\Psi_{1} \rightarrow 0} \mathbf{A}^{-1}=\left[\begin{array}{cccc}
1 / 2 & 1 / 2 & 0 & 0 \\
1 / 2 & 1 / 2 & 0 & 0 \\
0 & 0 & 1 & 0 \\
0 & 0 & 0 & 0
\end{array}\right]
$$

The return-mapping procedure is then given by

$$
\boldsymbol{\sigma}^{(i+1)}=\left[\begin{array}{cccc}
1 / 2 & 1 / 2 & 0 & 0 \\
1 / 2 & 1 / 2 & 0 & 0 \\
0 & 0 & 1 & 0 \\
0 & 0 & 0 & 0
\end{array}\right]\left\{\boldsymbol{\sigma}^{\mathrm{E}}-1 / 2 \Delta \lambda_{1} \mathbf{D}_{\mathrm{e}} \pi_{1}\right\}
$$

The second possible situation which may cause numerical problems is the situation where the final stress is at the apex of the Drucker-Prager yield, i.e. $\Psi_{2}=0$. Because the tensile stresses are bounded by the tensile strength, i.e. via the Rankine criterion, this situation is not likely to occur. More likely to occur is the situation where the equivalent stress $\bar{\sigma}_{2}$ is reduced to zero. This implies that the apex has been translated to the origin of the stress space and, consequently, the factor $\Psi_{2}$ is equal to zero. However, this situation is only possible if for the Rankine yield criterion the equivalent stress also equals zero and consequently, $\Psi_{1}=0$. The limit of the mapping matrix is then given by

$$
\lim _{\substack{\Psi_{1} \rightarrow 0 \\
\Psi_{2} \rightarrow 0}} A^{-1}=\left[\begin{array}{cccc}
1 / 3 & 1 / 3 & 1 / 3 & 0 \\
1 / 3 & 1 / 3 & 1 / 3 & 0 \\
1 / 3 & 1 / 3 & 1 / 3 & 0 \\
0 & 0 & 0 & 0
\end{array}\right]
$$

and the return-mapping procedure results in 


$$
\boldsymbol{\sigma}^{(i+1)}=\left[\begin{array}{cccc}
1 / 3 & 1 / 3 & 1 / 3 & 0 \\
1 / 3 & 1 / 3 & 1 / 3 & 0 \\
1 / 3 & 1 / 3 & 1 / 3 & 0 \\
0 & 0 & 0 & 0
\end{array}\right]\left\{\boldsymbol{\sigma}^{\mathrm{E}}-1 / 2 \Delta \lambda_{1} \mathbf{D}_{\mathrm{e}} \pi_{1}-\alpha_{g} \Delta \lambda_{2} \mathbf{D}_{\mathrm{e}} \pi_{2}\right\}
$$

\section{CONSISTENT LINEARIZATION OF THE STRESS UPDATE}

The set of non-linear equations that follow from the finite element discretization will be solved using the Newton-Raphson method. The non-linear problem is then linearized in a sequence of iterations until the problem is converged. The linearization of the equations results in the tangent stiffness matrix which plays an important role in the performance and robustness of the Newton-Raphson method. As has been argued by Simo and Taylor (1985), the quadratic convergence of Newton's method depends crucially on the consistent linearization of the stress resulting from the return-mapping algorithm to set up the tangent stiffness. The consistent tangent stiffness matrix for the Newton-Raphson procedure is most conveniently derived from the updated stress at iteration $(i+1)$

$$
\sigma^{(i+1)}=\mathbf{D}_{\mathbf{e}}\left\{\varepsilon^{(i+1)}-{ }^{t} \varepsilon_{\mathrm{c}}-\sum_{j=1}^{2} \Delta \lambda_{j}^{(i+1)} \partial_{\sigma} g_{j}\right\}
$$

The total derivative then reads

$$
\mathrm{d} \boldsymbol{\sigma}^{(i+1)}=\mathbf{D}_{\mathrm{e}}\left\{\mathrm{d} \boldsymbol{\varepsilon}^{(i+1)}-\sum_{j=1}^{2}\left(c_{j} \mathrm{~d} \lambda_{j} \partial_{\sigma} g_{j}+\Delta \lambda_{j}^{(i+1)} \partial_{\sigma \sigma}^{2} g_{j} \mathrm{~d} \sigma^{(i+1)}\right)\right\}
$$

which is written as

$$
\mathrm{d} \boldsymbol{\sigma}^{(i+1)}=\mathbf{H}\left\{\mathrm{d} \boldsymbol{\varepsilon}^{(i+1)}-\sum_{j=1}^{2} c_{j} \mathrm{~d} \lambda_{j} \partial_{\sigma} g_{j}\right\}
$$

where the modified stiffness matrix $\mathbf{H}$ is given by

$$
\mathbf{H}=\left[\mathbf{C}_{\mathrm{e}}+\Delta \lambda_{1}^{(i+1)} \partial_{\sigma \sigma}^{2} f_{1}+\Delta \lambda_{2}^{(i+1)} \partial_{\sigma \sigma}^{2} g_{2}\right]^{-1}
$$

Applying the general formulation of Riggs and Powell (1990), the tangent stiffness matrix is written for the present case as

$$
\mathrm{d} \boldsymbol{\sigma}^{(i+1)}=\mathbf{H}\left\{\mathrm{d} \boldsymbol{\varepsilon}^{(i+1)}-\mathbf{U d} \boldsymbol{\lambda}\right\}
$$

with the matrix $\mathbf{U}$ given by

$$
\mathbf{U}=\left[c_{1} \partial_{\sigma} f_{1}, c_{2} \partial_{\sigma} g_{2}\right]
$$

and the vector $\mathrm{d} \lambda=\left\{\mathrm{d} \lambda_{1}, \mathrm{~d} \lambda_{2}\right\}^{\mathrm{T}}$ collecting the plastic multipliers. The consistency conditions, $\dot{f}_{j}=0$, for the active yield surfaces give the relations for the inelastic multipliers

$$
c_{j}\left(\partial_{\sigma} f_{j}^{\mathrm{T}} \mathrm{d} \boldsymbol{\sigma}+\partial_{q} f_{j}^{\mathrm{T}} \mathrm{d} \mathbf{q}\right)+\left(1-c_{j}\right) \mathrm{d} \lambda_{j}=0 .
$$
Substituting the relation $\mathrm{d} q=\sum_{j=1}^{2} c_{j} \mathrm{~d} \lambda_{j} \mathbf{h}_{j}$ allows recasting of the consistency conditions in
the following format 


$$
\mathrm{d} \lambda=\mathbf{E}^{-1} \mathbf{V}^{\mathrm{T}} \mathrm{d} \boldsymbol{\sigma}
$$

with

$$
E_{i j}=-c_{i} c_{j} \partial_{q} f_{i}^{\mathrm{T}} \mathbf{h}_{j}-\left(1-c_{i}\right) \delta_{i j}
$$

where $\delta_{i j}$ is the Kronecker delta (no summation implied). The matrix $\mathbf{V}$ is given by

$$
\mathbf{V}^{\mathrm{T}}=\left[\begin{array}{l}
c_{1} \partial_{\sigma} f_{1}^{\mathrm{T}} \\
c_{2} \partial_{\sigma} f_{2}^{\mathrm{T}}
\end{array}\right]
$$

Substituting eqn (55) into eqn (52) results in

$$
\left[\mathbf{H}^{-1}+\mathbf{U} \mathbf{E}^{-1} \mathbf{V}^{\mathrm{T}}\right] \mathrm{d} \boldsymbol{\sigma}^{(i+1)}=\mathrm{d} \boldsymbol{\varepsilon}^{(i+1)} .
$$

The consistent tangent stiffness matrix can then be calculated with the application of the Sherman-Morrison-Woodbury formula :

$$
\mathrm{d} \boldsymbol{\sigma}^{(i+1)}=\left[\mathbf{H}-\mathbf{H} \mathbf{U}\left(\mathbf{E}+\mathbf{V}^{\mathrm{T}} \mathbf{H} \mathbf{U}\right)^{-1} \mathbf{V}^{\mathrm{T}} \mathbf{H}\right] \mathrm{d} \boldsymbol{\varepsilon}^{(i+1)} .
$$

It is noted that if the yield functions are coupled through the hardening functions given in eqn (16), or if $\alpha_{f} \neq \alpha_{g}$, the tangent stiffness matrix is non-symmetric.

\section{APPLICATION TO PLAIN CONCRETE}

The first application concerns a cube splitting test which is often used as an indirect test for determining the tensile strength of concrete. This example has been chosen to analyze the capability of the developed models to predict the failure mode in a tensioncompression test. The specimen which will be analyzed is a cube with a side of $150 \mathrm{~mm}$. Only half of the specimen has been discretized because of symmetry conditions, with two different discretizations in order to show the mesh-insensitivity. The two different meshes are shown in Fig. 5, the first discretization with $21 \times 9$ cross-diagonal constant strain triangles with the total number of elements equal to 756 . The second discretization concerns a refinement with a factor nine, resulting in a total number of elements equal to 6804 . The loading platen is assumed to be rigid and has been modeled by a equal vertical constraint of the appropriate nodes. The analyses have been performed using a constrained NewtonRaphson iteration with line-searches; for details about this advanced solution strategy see Feenstra (1993).

The material which is considered is a concrete with a mean value of the compressive strength $f_{\mathrm{cm}}=35 \mathrm{MPa}$ and a maximum aggregate size of $d_{\max }=8 \mathrm{~mm}$. According to CEBFIP model code regulations (CEB-FIP model code, 1990) the following material parameters can be assigned: Young's modulus $E_{\mathrm{c}}=32,710 \mathrm{MPa}$, Poisson's ratio $v=0.15$, tensile strength $f_{\mathrm{ct}, \mathrm{m}}=2.7 \mathrm{MPa}$ and a tensile fracture energy $G_{\mathrm{f}}=0.06 \mathrm{~N} \mathrm{~mm}^{-1}$. The CEB-FIP model code does not supply a rclationship for the compressive fracture encrgy. This parameter has been chosen as $G_{\mathrm{c}}=5.0 \mathrm{~N} \mathrm{~mm}^{-1}$.

The load versus the displacement of the loading platen is depicted in Fig. 6, from which two different failure mechanisms can be distinguished. First, a splitting crack is formed at a load level of approximately $30 \mathrm{~N} \mathrm{~mm}^{-2}$, which is attended with a reduction of the load. When the crack is fully developed the load starts to increase again, leading to a collapse mechanism which is governed by a compressive failure.

The deformations are shown in Fig. 7 for the coarse mesh and in Fig. 8 for the refined mesh. Both meshes show the same pattern, with the splitting crack occurring in the middle of the specimen just after the first local maximum in the load-displacement diagram, Figs 7(a) and 8(a). The final deformations are shown in Figs 7(b) and 8(b) for the coarse and 


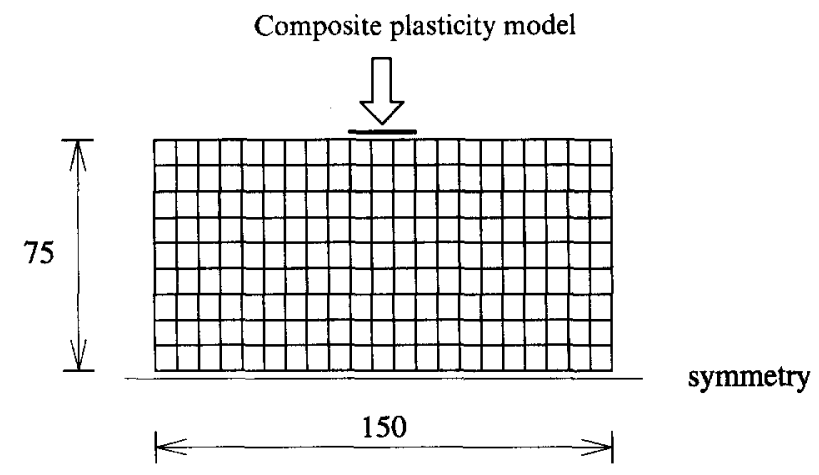

(a)

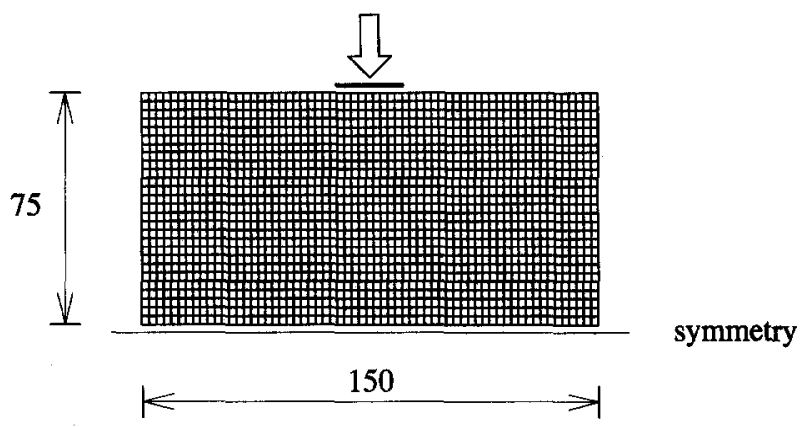

(b)

Fig. 5. Finite element meshes for a cube splitting test. Crossed triangular elements : (a) coarse mesh ; (b) refined mesh.

the refined mesh, respectively. In both figures the final failure mode is a combination of the tensile splitting crack and the compressive failure mode which results in a wedge which is pushed into the specimen, separating the two parts.

The failure mechanism can also be explained by the distribution of the internal damage parameters, $\kappa_{1}$ and $\kappa_{2}$; see Fig. $9(\mathrm{a}, \mathrm{b})$ for the coarse mesh. The magnitude of the internal parameters is scaled to the highest value which is shown as solid black. The distribution just after the first peak load clearly shows the splitting crack in the middle of the specimen

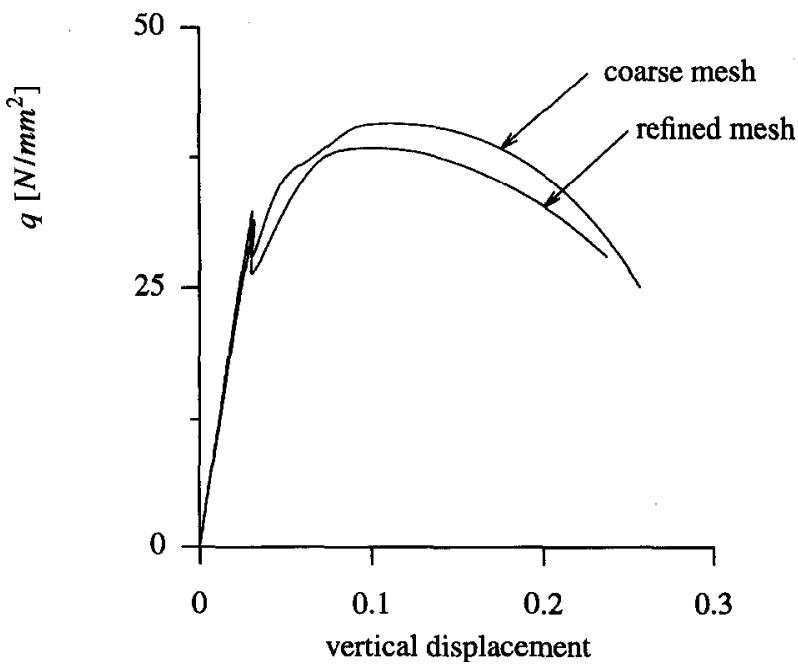

Fig. 6. Cube splitting test. Load-deformation diagrams. 


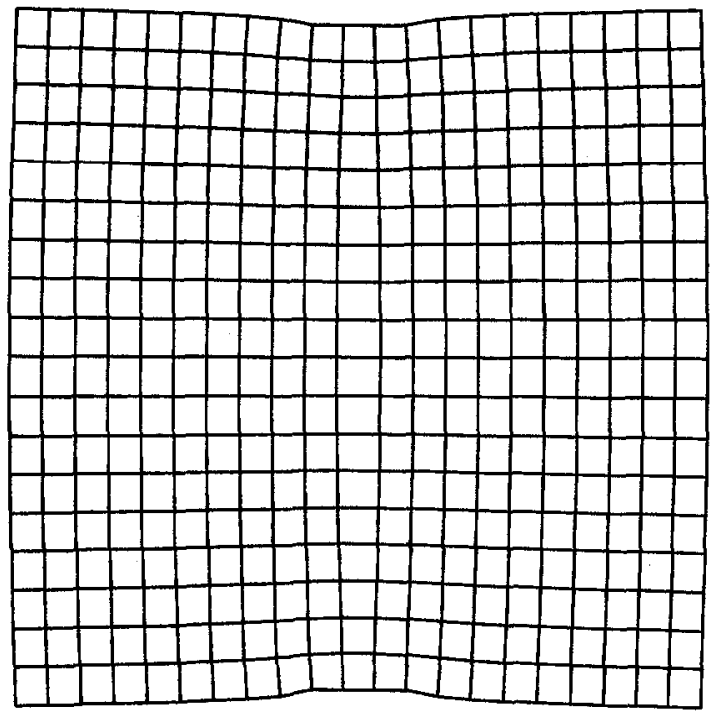

(a)

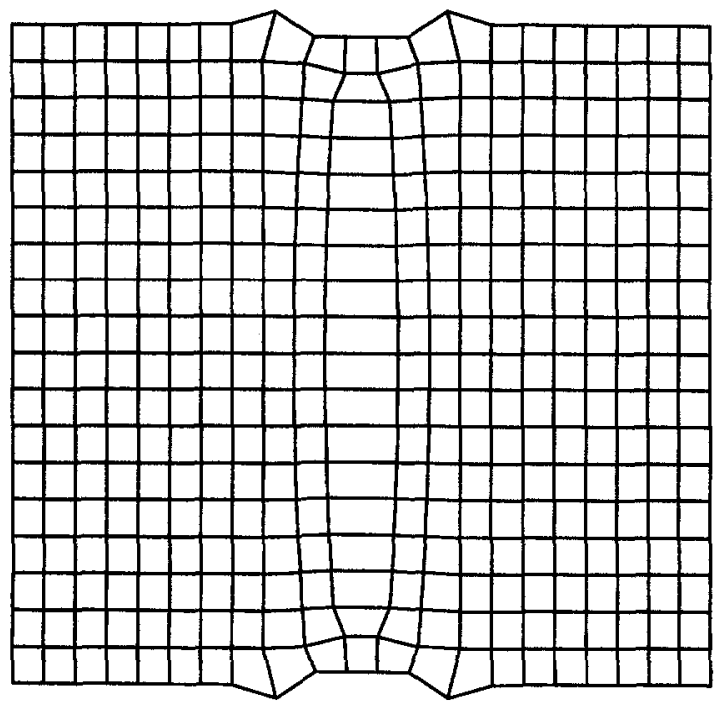

(b)

Fig. 7. Cube splitting test coarse mesh. Deformations : (a) after first peak load (deformation $\times 100$ ) ; (b) at final load (deformation $\times 10$ ).

and the more distributed damage parameter related to the compressive regime. In the final stage also, the distribution of $\kappa_{2}$ localizes in a wedge-like shape under the loading platen. The results for the refined mesh, Fig. 10(a, b), show this transition even more clearly.

\section{APPLICATION TO REINFORCED CONCRETE}

The analysis of shear wall panels is a good example of the possible application of the composite plasticity model to reinforced concrete. The stress state in the panels can be considered to be in tension-compression. The panel which will be presented in this study has been tested at the E.T.H. Zürich by Maier and Thürlimann (1985) and has been analyzed before by Wang et al. (1990). The constitutive model which was used in that study is a combination of a fixed crack model to describe the tensile stress state and a MohrCoulomb plasticity model to describe the compressive stress states (de Borst and Nauta, 


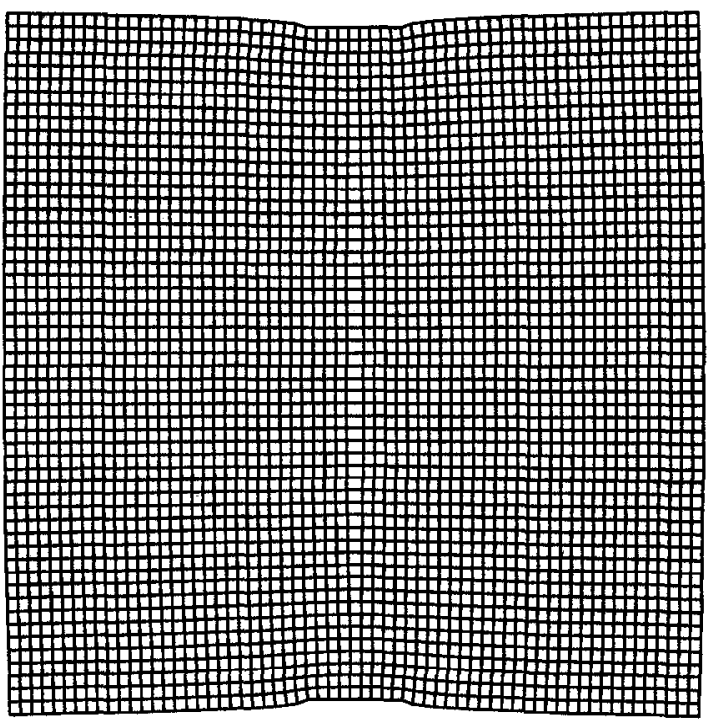

(a)

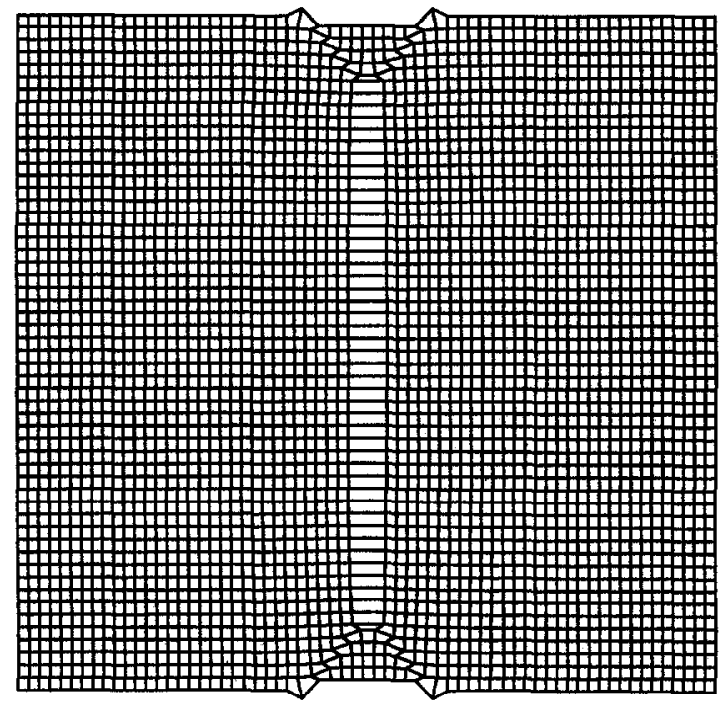

(b)

Fig. 8. Cube splitting test refined mesh. Deformations : (a) after first peak load (deformation $\times 100$ ); (b) at final load (deformation $\times 10)$.

1985). However, the combination of cracking and plasticity resulted in convergence problems if a large region existed in which both the cracking and the plasticity model became active. These numerical problems were solved by defining two areas in which either only the cracking model or only the plasticity model could become active. The solutions which were obtained with this approach are in good agreement with the experimental results which indicates that the method is rather effective. The arbitrariness of defining the regions a priori is a major drawback of this method, and the analyses with the combined yield surface presented here show that convergence problems are avoided if a stable algorithm is used.

The panel which will be analyzed is panel S2 of the experimental program of Maier and Thürlimann (1985). The panel is initially loaded by a vertical compressive force, and then loaded by a horizontal force until the experiment becomes unstable and the failure load has been reached. In the experimental set-up, the panels were supported on a base 


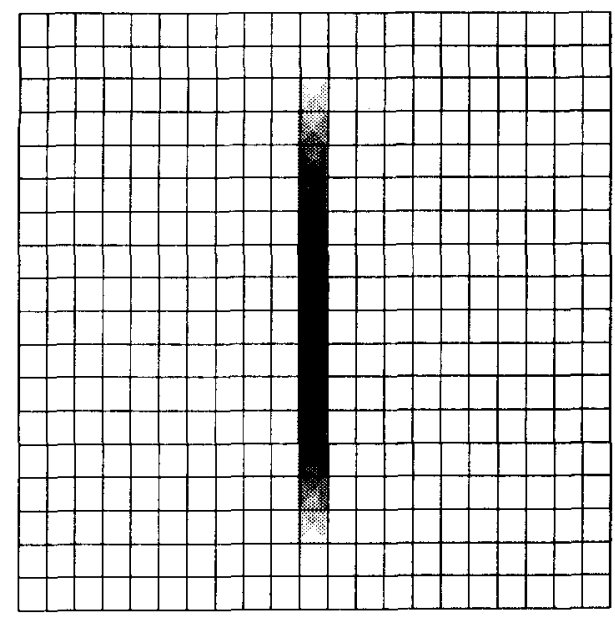

(a)

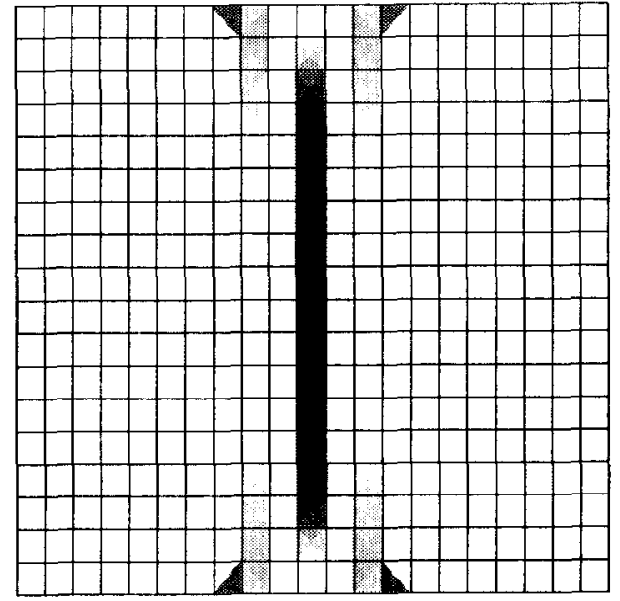

(c)

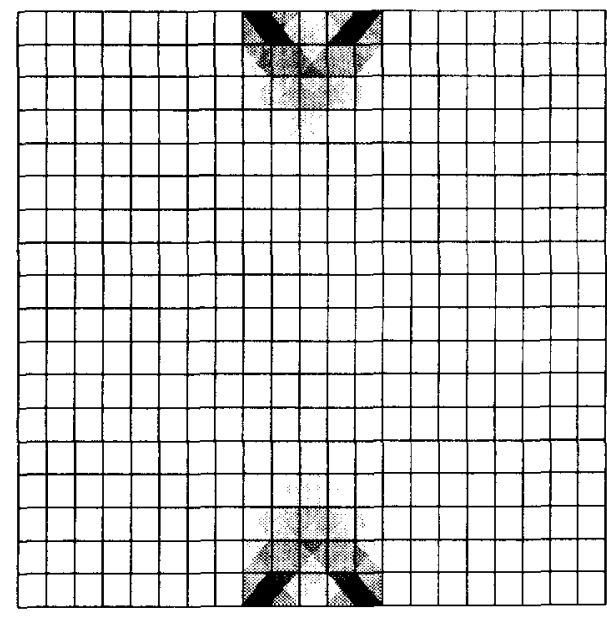

(b)

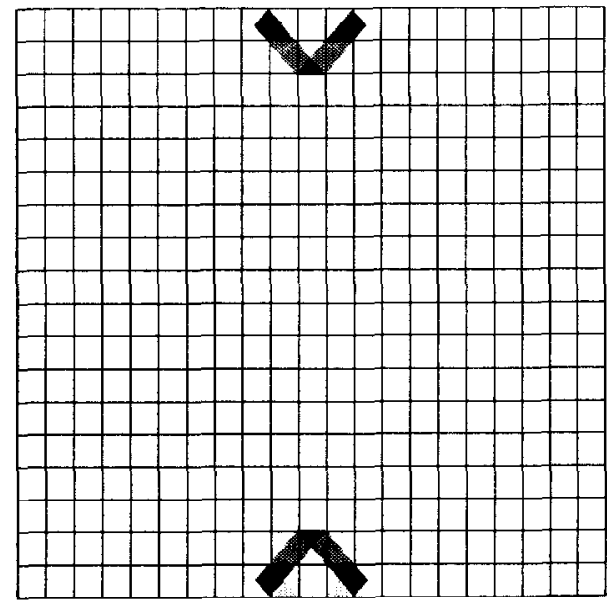

(d)

Fig. 9. Cube splitting test coarse mesh. Distribution of the internal parameters : (a) $\kappa_{1}$ after first peak load; (b) $\kappa_{2}$ after first peak load; (c) $\kappa_{1}$ at final load; (d) $\kappa_{2}$ at final load.

block and loaded through a thick top slab, Fig. 11(a). In the finite element discretization, the top slab has been modeled with linear-elastic elements without reinforcement, whereas the supporting block has been replaced by fixed supports in the $x$-and $y$-directions. The finite element discretization is depicted in Fig. 11(b) with quadratic plane-stress elements with a nine-point Gaussian integration for both the reinforcement and the element. The reinforcement is applied by reinforcing grids in two directions with a diameter of $8 \mathrm{~mm}$ and a clear cover of $10 \mathrm{~mm}$. The reinforcement ratios in the web of the panel are equal to 0.0103 and 0.0116 for the $x$ - and $y$-directions, respectively. The reinforcement ratio in the flange of the panel is equal to 0.0116 .

The material properties have been averaged from the experimental data provided by Thürlimann and Maier (1985) with a reduction of the compressive strength of $20 \%$, which results in a mean compressive strength $f_{\mathrm{cm}}=27.5 \mathrm{MPa}$. According to CEB-FIP model code regulations (CEB-FIP model code, 1990) the following material parameters have been assigned: Young's modulus $E_{\mathrm{c}}=30,000 \mathrm{MPa}$, Poisson's ratio $v=0.15$, tensile strength $f_{\mathrm{ct}, \mathrm{m}}=2.2 \mathrm{MPa}$ and a tensile fracture energy $G_{\mathrm{f}}=0.07 \mathrm{~N} \mathrm{~mm}^{-1}$. The compressive fracture energy has been chosen as $G_{\mathrm{c}}=50.0 \mathrm{~N} \mathrm{~mm}^{-1}$.

The horizontal and vertical load have been applied as a uniformly distributed element load as indicated in Fig. 11(b). The horizontal displacement $u_{\mathrm{h}}$ of the top slab has been 


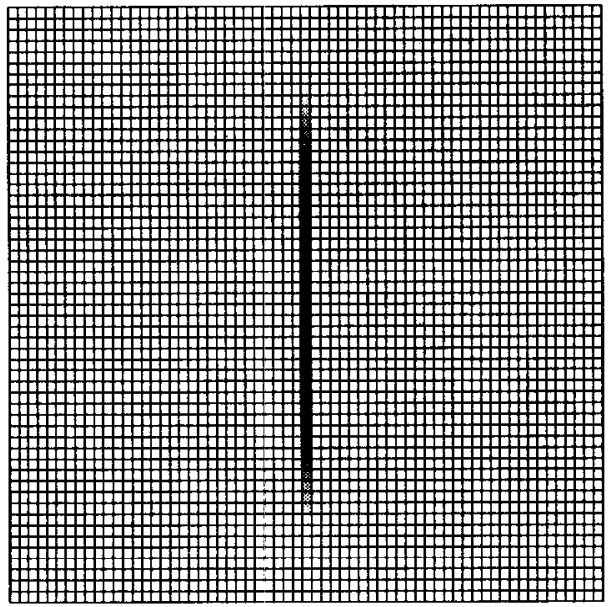

(a)

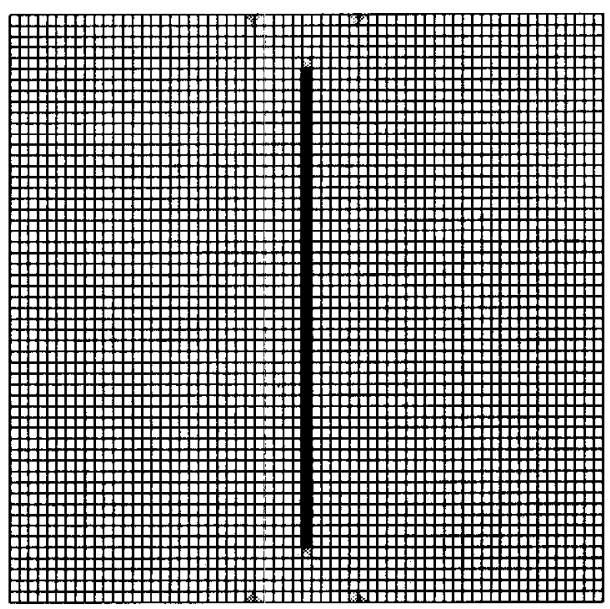

(c)

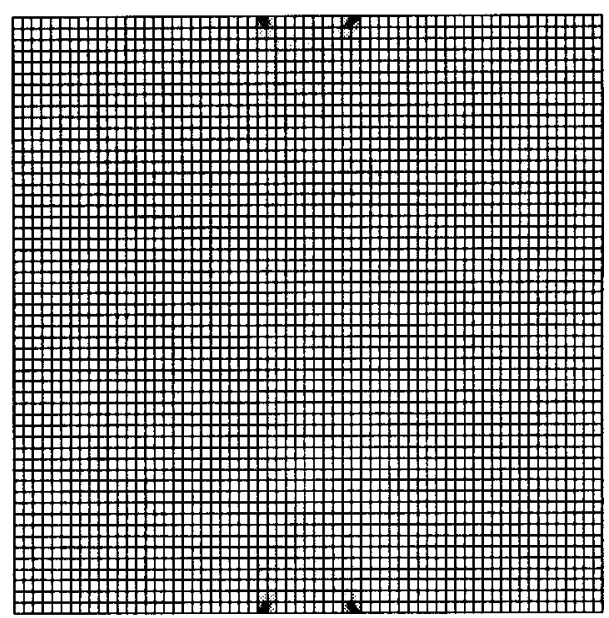

(b)

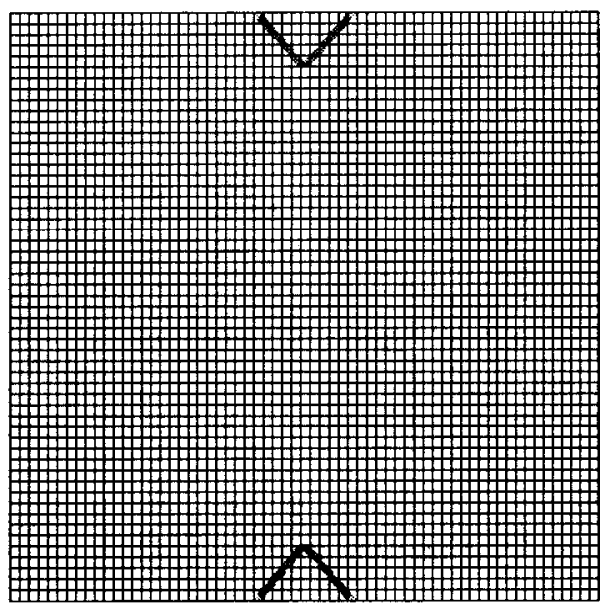

(d)

Fig. 10. Cube splitting test refined mesh. Distribution of the internal parameters : (a) $\kappa_{1}$ after first peak load; (b) $\kappa_{2}$ after first peak load; (c) $\kappa_{1}$ at final load; (d) $\kappa_{2}$ at final load.

monitored and compared with the experimental load-displacement curves. The NewtonRaphson equilibrium iteration has been applied with an indirect displacement control method. The displacement in the horizontal direction $u_{\mathrm{h}}$ has been chosen as the active degree-of-freedom with load steps of approximately $0.1 \mathrm{~mm}$. With this solution technique, converged solutions could be obtained in the complete loading regime.

Panel S2 is subjected to an initial vertical load of $1653 \mathrm{kN}$ ( $\equiv 10.0 \mathrm{MPa}$ ) which results in an initial horizontal displacement of $0.29 \mathrm{~mm}$ in the experiment. The calculated initial displacement is equal to $-54 \times 10^{-6} \mathrm{~mm}$ which indicates a possible eccentricity in the experimental set-up. After the initial vertical load, the horizontal load is applied using indirect displacement control. The load-displacement diagram is shown in Fig. 12, which shows a reasonable agreement between experimental and calculated response for both the coarse and the refined meshes. The experimental failure mechanism was rather explosive and caused a complete loss of load-carrying capacity (Maier and Thürlimann, 1985), which can be explained by the brittle behavior of the panel after maximum load, Fig. 12.

Further results of the analysis are shown in Figs 13-15 at the final load. Figure 13 shows the deformation of the panel for both meshes. Apparently, the failure mechanism is highly localized near the supports. The distribution of the internal parameters $\kappa_{1}$ and $\kappa_{2}$ at the final load is shown in Figs 14 and 15 for the coarse mesh and the refined mesh, 

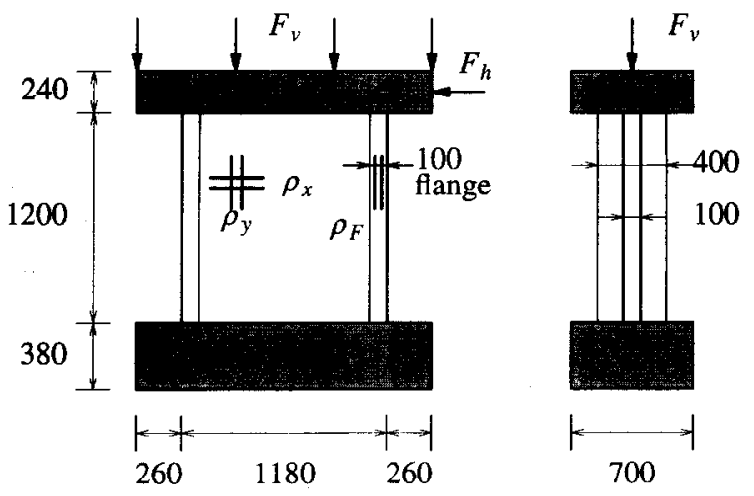

(a)

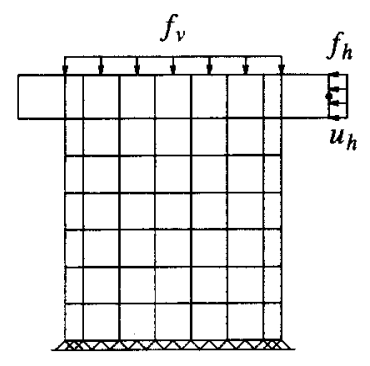

(b)

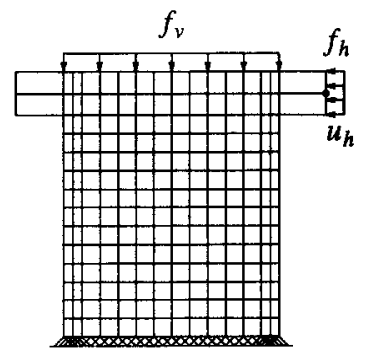

Fig. 11. Experimental set-up and finite element discretization of shear wall panel S2 (Maier and Thürlimann, 1985).

respectively. These figures show that the panel is densely cracked with plastic points in the bottom-left corner of the panel and in the compressive flange.

\section{CONCLUDING REMARKS}

A composite yield function has been developed with particular reference to the proper modeling of tension-compression biaxial stress states in concrete structures. Structural parts that are stressed under this condition often act as an initiation point for explosive crack propagation. The main advantage of the implicit algorithm that is based upon this notion of a composite yield function is its robustness. This has been shown in this contribution for some typical unreinforced concrete structures, and for a typical "difficult" case in reinforced concrete like the shear wall.

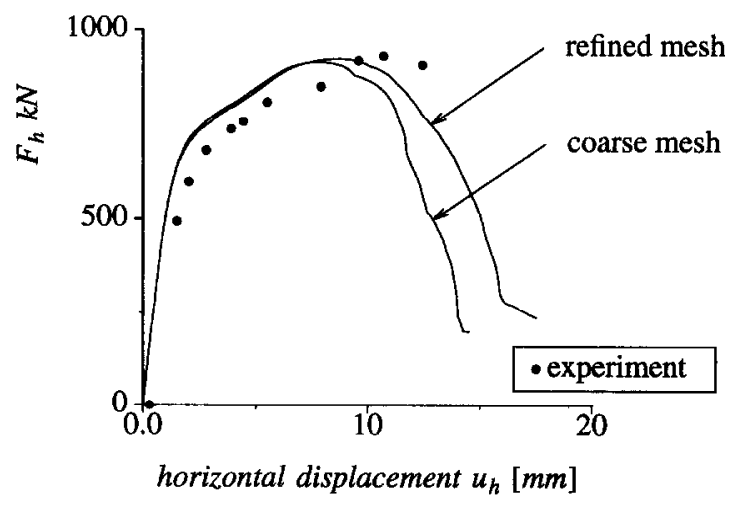

Fig. 12. Panel S2. Load-displacement diagrams. 


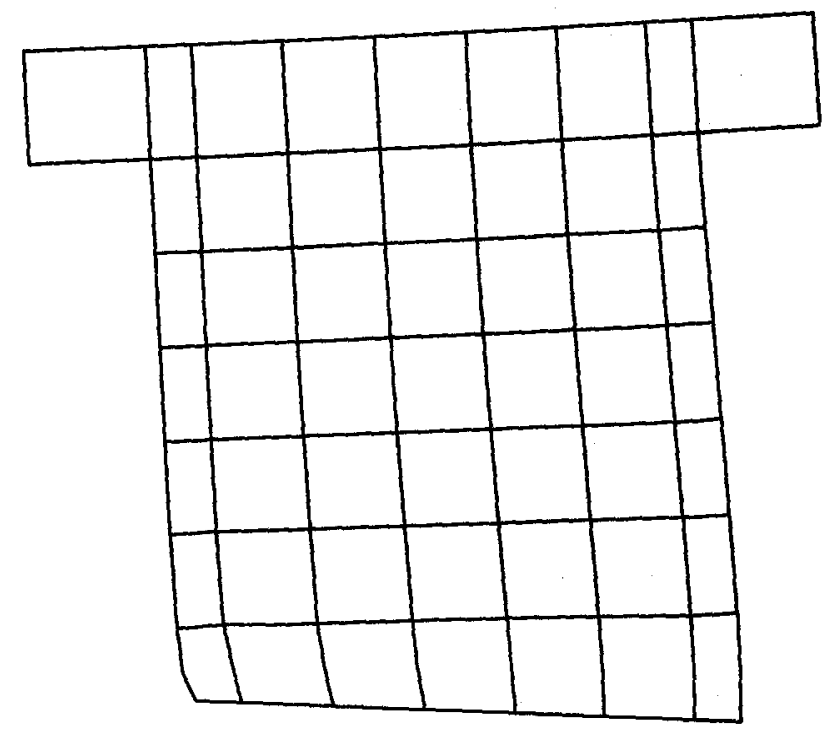

(a)

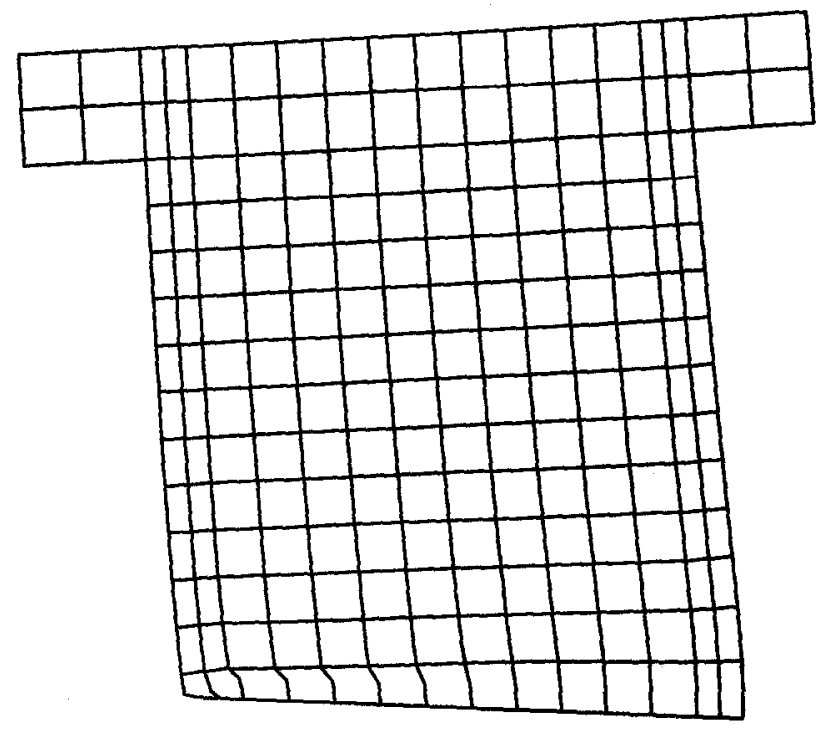

(b)

Fig. 13. Panel S2. Deformations at final load (deformation $\times 10$ ). 


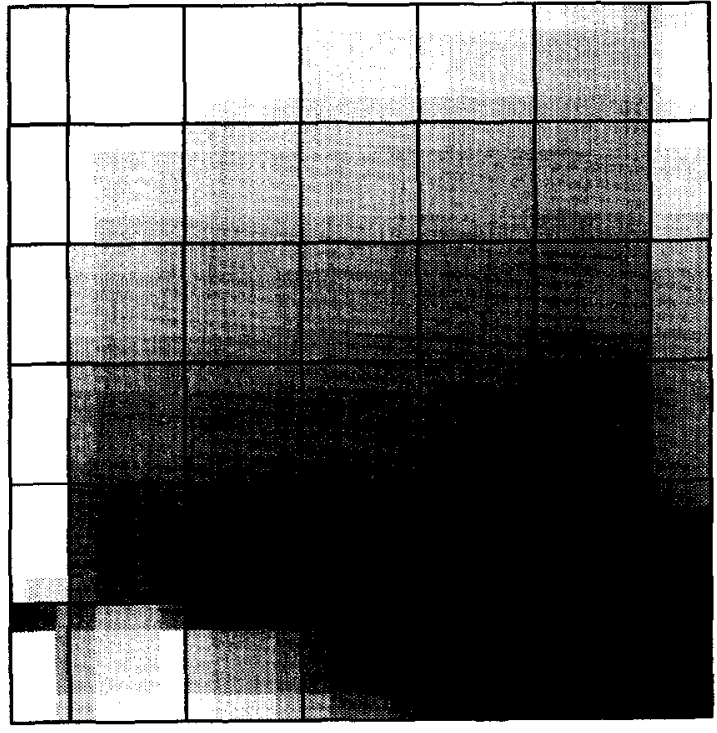

(a)

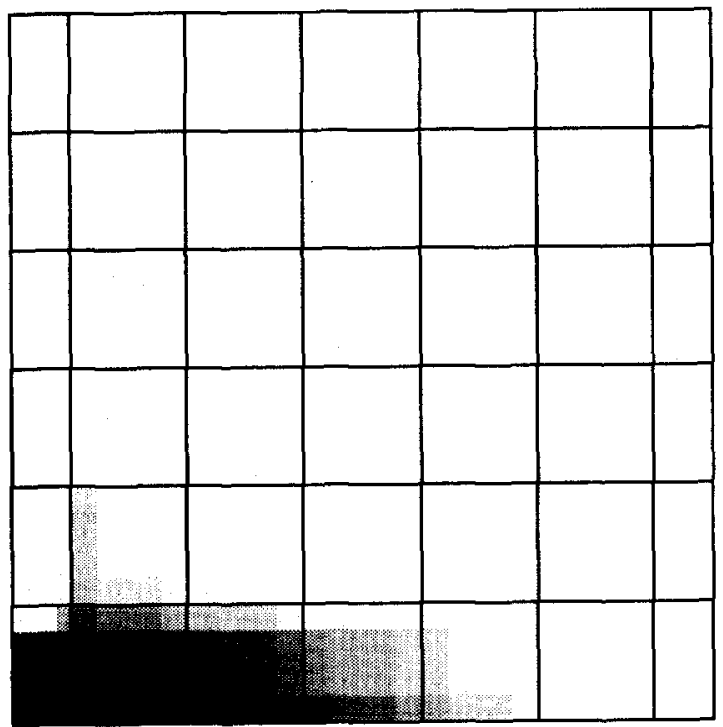

(b)

Fig. 14. Panel S2, coarse mesh. Distribution of the internal parameters: (a) $\kappa_{1}$ at final load; (b) $\kappa_{2}$ at final load. 


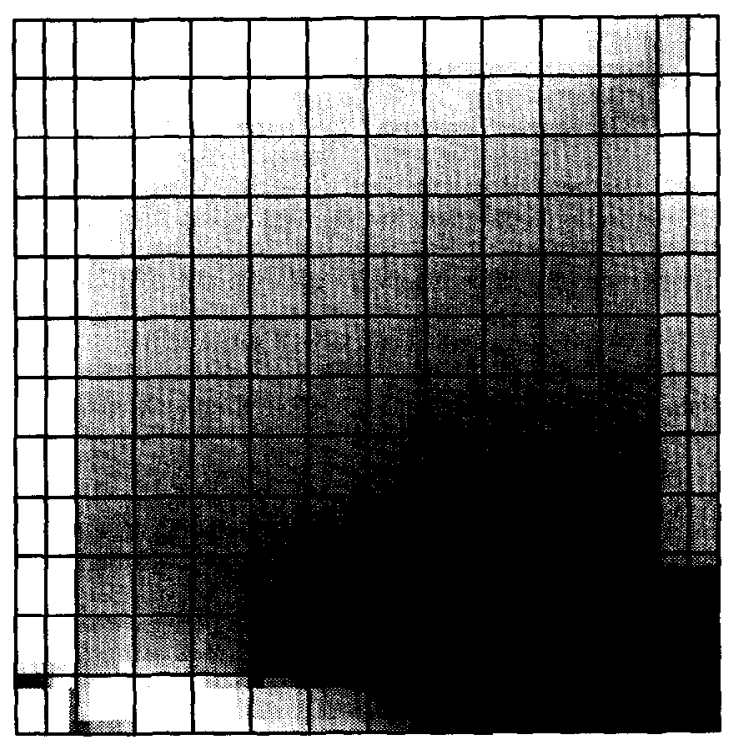

(a)

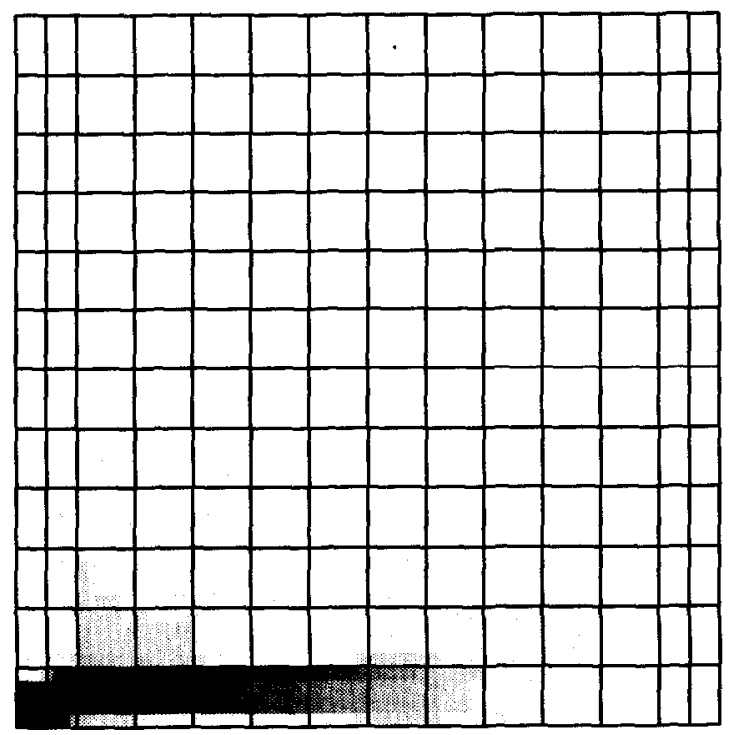

(b)

Fig. 15. Panel S2, refined mesh. Distribution of the internal parameters : (a) $\kappa_{1}$ at final load ; (b) $\kappa_{\text {, }}$ at final load. 
Acknowledgements-The calculations have been carried out with a pilot version of the DIANA finite element code. Financial support by the Commission of the European Communities under the Brite-EuRam programme (project BE-3275) and by the Netherlands Technology Foundation (grant DCT72.1405) is gratefully acknowledged.

This publication has been partly prepared during a stay of the first author at Stanford University, supported by a NATO-Science Fellowship.

\section{REFERENCES}

Bažant, Z. P. and Oh, B. H. (1983). Crack band theory for fracture of concrete. Mater. Structures, RILEM 93, $155-177$.

Borst, R. de (1986). Non-linear analysis of frictional materials. Dissertation, Delft University of Technology.

Borst, R. de (1991). The zero-normal stress condition in plane-stress and shell elasto-plasticity. Comm. Appl. Numer. Meth. 7, 29-33.

Borst, R. de (1993). A generalisation of $J_{2}$-flow theory for polar continua. Comp. Meth. Appl. Mech. Engng 103, $347-362$.

Borst, R. de and Nauta, P. (1985). Non-orthogonal cracks in a smeared finite element model. Engng Comput. 2, $35-46$.

Borst, R. de, Sluys, L. J., Pamin, J. and Mühlhaus, H.-B. (1993). Fundamental issues in finite element analysis of localization of deformation. Engng Comput. 10, 99-121.

Buyukozturk, O. (1977). Nonlinear analysis of reinforced concrete structures. Comput. Structures 7, 149-156.

CEB-FIP (1990). Model code 1990. Bulletin d'Information, CEB, Lausanne.

Chen, A. C. T. and Chen W. F. (1975). Constitutive relations for concrete J. Engng Mech., ASCE 101, 465-481.

Crisfield, M. A. (1982). Accelerated solution techniques and concrete cracking. Comp. Meth. Appl. Mech. Engng 33, 858-607.

Crisfield, M. A. and Wills, J. (1989). Analysis of R/C panels using different concrete models. J. Engng Mech., ASCE 115, 578-597.

Dennis, J. E. and Schnabel, R. B. (1983). Numerical Methods for Unconstrained Optimization and Nonlinear Equations. Prentice IIall, NJ.

Feenstra, P. H. (1993). Computational aspects of biaxial stress in plain and reinforced concrete. Dissertation, Delft University of Technology.

Hordijk, D. A. (1991). Local approach to fatigue of conerete. Dissertation, Delft University of Technology.

Koiter, W. T. (1953). Stress-strain relations, uniqueness and variational theorems for elastic-plastic materials with a singular yield surface. Q. Appl. Math. 11, 350-354.

Kupfer, H. B. and Gerstle, K. H. (1973). Behavior of concrete under biaxial stresses. J. Engng Mech., ASCE 99, 853-866.

Maier, J. and Thürlimann, B. (1985). Bruchversuche an Stahlbetonscheiben. Report 8003-1, Eidgenössische Technische Hochschule, Zürich.

Mier, J. G. M. van (1984). Strain-softening of concrete under multiaxial loading conditions. Dissertation, Eindhoven University of Technology.

Murray, D. W., Chitnuyanondh, I., Rijab-Agha, K. Y. and Wong, C. (1979). A concrete plasticity theory for biaxial stress analysis J. Engng Mech., ASCE 105, 989-1106.

Oliver, J. (1989). A consistent characteristic length for smeared crack models. Int. J. Numer. Meth. Engng 28, $461-474$.

Ortiz, M. and Popov, E. P. (1985). Accuracy and stability of integration algorithms for elastoplastic constitutive relations. Int. J. Numer. Meth. Engng 21, 1561-1576.

Pietruszczak, S. and Mróz, Z. (1981). Finite element analysis of deformation of strain-softening materials. Int. $J$. Numer. Meth. Engng 17, 327-334.

Pramono, E. and Willam, K. J. (1989). Implicit integration of composite yield surfaces with corners. Engng Comput. 7, 186-197.

Rashid, Y. R. (1968). Analysis of prestressed concrete pressure vessels. Nucl. Engng Design 7, 334-344.

Riggs, H. R. and Powell, G. H. (1990). Tangent constitutive matrices for inelastic finite element analyses. Int. J. Numer. Meth. Engng 29, 1193-1203.

Rots, J. G. (1988). Computational modeling of concrete fracture. Dissertation, Delft University of Technology.

Rüsch, H. and Hilsdorf, H. (1963). Verformungseigenschaften von Beton unter zentrischen Zugspannungen (in German). Bericht Nr 44, Materialprüfungsamt für das Bauwesen der Technischen Hochschule München.

Schellekens, J. C. J. and de Borst, R. (1991). The use of the Hoffman yield criterion in finite element analysis of anisotropic composites. Comput. Structures 37, 1087-1096.

Simo, J. C., Kennedy, J. G. and Govindjee, S. (1988). Non-smooth multisurface plasticity and viscoplasticity. Loading/unloading conditions and numerical algorithms. Int. J. Numer. Meth. Engng 26, 2161-2185.

Simo, J. C. and Taylor, R. L. (1985). Consistent tangent operators for rate-independent elastoplasticity. Comp. Meth. Appl. Mech. Engng 48, 101-118.

Sluys, L. J. (1992). Wave propagation, localisation and dispersion in softening solids. Dissertation, Delft University of Technology.

Vonk, R. A. (1992). Softening of concrete loaded in compression. Dissertation, Eindhoven University of Technology.

Wang, Q. B., Van der Vorm, P. L. J. and Blaauwendraad, J. (1990). Failure of reinforced concrete panels-How accurate the models must be? In Computer Aided Analysis and Design of Concrete Structures (Edited by N. Bićanić and HI. A. Mang), pp. 153-163. Pineridge Press, Swansea.

Willam, K. J. (1984). Experimental and computational aspects of concrete fracture. In Computer Aided Analysis and Design of Concrete Structures (Edited by N. Bićanić et al.), pp. 33-70. Pineridge Press.

Willam, K. J., Hurlbut, B. and Sture, S. (1986) Experimental and constitutive aspects of concrete failure. In Finite Element Analysis of Reinforced Concrete Structures (Edited by C. Meyer and H. Okamura), ASCE, NY, pp. 226-245.

Willam, K. J. and Warnke, E. P. (1975) Constitutive model for triaxial behavior of concrete. In Colloquium on Concrete Structures Subjected to Triaxial Stresses, IABSE Report, Vol. 19, JABSE, Hönggerbergs, pp. 1-30. 